\title{
CONSIDERACIONES SOBRE MODELOS DE AJUSTE EMPLEADOS EN LA DEMOGRAFÍA MATEMÁTICA ${ }^{1}$
}

\author{
A.LEJANDRo Mina V aldÉs \\ El Colegio de México
}

\section{Introducción}

Dentro de las investigaciones demográficas, en especial las que se refieren a la cuantificación de los fenómenos demográficos, tienen gran importancia los modelos de ajuste. Dichos modelos de ajuste sirven para corregir, suavizar o describir el comportamiento de dichos fenómenos. En general se desconoce o no se resalta el alcance del ajuste, es decir, se aplica el modelo, y en algunos casos se piensa que corrigió la información y lo que hizo fue únicamente suavizarla o describirla; el otro caso es cuando se cree que se suavizó el comportamiento del fenómeno y lo que realmente se hizo fue corregirlo.

El presente trabajo tiene la intención de presentar modelos que suavicen, corrijan y describan los fenómenos demográficos tales como la fecundidad, la nupcialidad y la mortalidad. Para el fenómeno fecundidad se trabaja con el polinomio o función de fecundidad de W. Brass y el modelo de fecundidad de A. J. Coale. En cuanto al fenómeno nupcialidad se toma, por una parte, el modelo bilogístico de A. Bocaz y por otra parte el modelo estándar de nupcialidad de A.J. Coale; finalmente con el fenómeno mortalidad se usa el sistema logito de W. Brass y la ley de Makeham.

Inicialmente se presentan cada uno de los modelos, mostrando los alcances y limitaciones de cada uno de ellos, tratando de resaltar los casos en que se está suavizando o describiendo el comportamiento del fenómeno demográfico, de los casos en donde se está corrigiendo

1 Este trabajo es una sintesis de la tesis presentada por el autor para obtener el grado de Matemático en la Facultad de Ciencias de la UNAM el 14 de agosto de 1981. 
la distribución observada del fenómeno; continuando con la presentación y comentarios de los ajustes obtenidos, para presentar finalmente conclusiones generales acerca de cada uno de los modelos de ajuste estudiados.

\section{Presentación de los modelos de ajuste}

A continuación se ofrece una descripción de cada uno de los modelos de ajuste estudiados, dando un ejemplo de su aplicación al final de cada presentación. En general, las gráficas que muestran la bondad de los ajustes no son presentadas en este apartado, sino en la presentación y comentario de los resultados obtenidos.

\subsection{El modelo de nupcialidad de A. J. Coale ${ }^{2}$}

E1 modelo teórico desarrollado por Ansley J. Coale surgió como resultado de las comprobaciones realizadas por él, al examinar en diferentes poblaciones el comportamiento de la nupcialidad por edad a través de las proporciones de no solteras y de las frecuencias de primeros matrimonios.

Dadas las proporciones de mujeres no solteras por años de edad para diferentes cohortes, Coale observó que las curvas construidas con esas proporciones difieren, entre sí en la medida en que la nupcialidad de las poblaciones en estudio presentan características diferentes en relación con la edad más baja de ingreso al matrimonio, el ritmo de aumento de ese ingreso desde la edad en que se inicia hasta la edad en que el número de ingresos es máximo, y la intensidad de là nupcialidad, representada por la proporción final; Coale observó que si las proporciones anteriores se presentaban a partir de un origen común, con una escala vertical ajustada convenientemente para cada población, de manera que la proporción de mujeres no solteras en las edades avanzadas sea igual a la unidad, y con una escala horizontal elegida de modo que la tasa media de ascenso de las curvas sea casi la misma, las curvas resultantes son prácticamente coincidentes.

Lo anterior quiere decir que las curvas originales tienen esencialmente la misma estructura, difiriendo únicamente en la edad en que comienza el ingreso al matrimonio, la tasa a la que aumenta el matrimonio y la proporción final de mujeres no solteras.

2 Ver Coale, A.J. "Age Patterns of Marriage". Population Studies, Vol. XXV, No. 2. pp. 193-214, julio, 1971. 
El que exista un patrón común de proporciones de mujeres no solteras, por edad, en diferentes poblaciones, implica que existe un patrón común de frecuencias de los primeros matrimonios en las poblaciones de referencia. Coale observó que si las curvas que representan esas frecuencias, calculadas a partir de los matrimonios registrados por años de edad, en poblaciones que tienen características diferentes en relación con la nupcialidad, se llevan a un origen común y con escalas horizontal y vertical convenientemente elegidas para cada población, las curvas resultantes también presentan una sorprendente similitud.

El que exista un patrón común de frecuencias de los primeros matrimonios implica un mismo patrón de riesgo de primer matrimonio, según la edad, que, de acuerdo con lo observado en las poblaciones estudiadas por Coale, parece ser independiente del tamaño del grupo de mujeres destinadas a permanecer solteras.

El patrón común de riesgo de primer matrimonio sugirió a Coale la posibilidad de construir una curva estándar que represente ese riesgo. La curva deberia ascender desde el origen, dado por la edad más temprana en que la cohorte comienza a contraer matrimonio, hasta un valor máximo, aproximadamente constante, cuando ya se ha casado la mayoría de las mujeres destinadas a no permanecer solteras. E] modelo estándar de riesgo de primer matrimonio se derivó a partir de los datos referentes a los matrimonios registrados en Suecia en 1865-1869, por años de edad, y ajustados por una doble exponencial.

La función propuesta por Coale es:

$$
\mathrm{r}_{\mathrm{S}}(\mathrm{x})=0.174 \mathrm{e}^{-4.411 \mathrm{e}^{-0.309 \mathrm{x}}}
$$

En donde $r_{S}(x)$ representa el riesgo estándar de nupcialidad, es decir, la probabilided de que una mujer soltera ingrese al estado matrimonial a la edad x de la escala horizontal ajustada.

En una cohorte en que los primeros matrinıonios comienzan a la edad (a) y para la cual la escala de la edad al casarse (eje de las abscisas) está reducida por un factor $\mathrm{K}$, el riesgo de matrimonio de las mujeres que han de casarse a la edad (a) es:

$$
r(a)=\frac{0.174}{K} e^{-4.411 e^{-\frac{0.309}{K}\left(a-a^{\circ}\right)}}
$$


Los tres parámetros que permiten caracterizar el comportamiento de la nupcialidad, mencionados anteriormente, se definen, en el modelo de Coale, de la siguiente manera:

$a^{\circ} \quad$ Representa la edad más joven de ingreso al primer matrimonio y corresponde al origen de la curva.

K. Corresponde al factor relacionado con la escala del tiempo durai,te el cual ocurren los primeros matrimonios, si en la curva estándar el intervalo entre el origen y el valor máximo es $\mathrm{x}$, para otra curva el intervalo es $\mathrm{K}_{\mathrm{x}}$. Por tanto el parámetro $\mathrm{K}$ es el factor que relaciona el ritmo de ingreso al primer matrimonio en una población, con el comportamiento de ese ingreso en la población que sirvió de base al modelo estándar. $\mathrm{El}$ valor de $\mathrm{K}$ siempre es positivo, si $\mathrm{K}$ es menor que uno, el ingreso al matrimonio en la población real ocurre a un ritmo más rápido que en el modelo; por el contrario, si $\mathrm{K}$ es mayor que uno, el ritmo es más lento que en el modelo.

C. Representa la producción de, mujeres no solteras a la edad en que las frecuencias de los primeros matrimonios es cercano a cero. Dicha edad depende, de $\mathrm{a}^{\circ}$ y principalmente de $\mathrm{K}$. Cuanto más bajo es el valor de $\mathrm{K}$, más rápido es el ritmo a que se producen los ingresos al primer matrimonio; lo que trae como consecuencia que el valor $\mathrm{C}$ se presente a una edad más joven.

Ahora bien, si $\mathrm{X}_{\mathrm{S}}$ representa la edad en la escala estándar $\mathrm{g}_{\mathbf{S}}\left(\mathrm{X}_{\mathrm{S}}\right)$ será la frecuencia con que ocurren los primeros matrimonios a la edad $\mathrm{X}_{\mathrm{S}} ; \mathrm{G}_{\mathrm{S}}\left(\mathrm{X}_{\mathrm{S}}\right)$ la proporción estándar de mujeres no solteras a la edad $\mathrm{X}_{\mathrm{S}}$, y $Z_{S}\left(X_{S}\right)$ el número de año-personas vividas como no solteras hasta la edad $\mathrm{X}_{\mathrm{S}}$, en una cohorte no expuesta a los riesgos de mortalidad y migración, así se tiene que:

$$
\begin{aligned}
& G_{s}\left(X_{S}\right)=\int_{0}^{X_{s}^{\prime}} g_{s}\left(X_{s}\right) d X_{s} \\
& Z_{S}\left(X_{S}\right)=\int_{0}^{X_{S}^{\prime}} G_{S}\left(X_{S}\right) d X_{S} \quad \text { siendo } 0<X_{S}<X_{S}^{\prime}
\end{aligned}
$$




\subsubsection{Ejemplo de la aplicación del modelo de nupcialidad de Coale:}

Para aplicar este modelo se requiere la información, por edad individual, de las proporciones de primeros matrimonios (para fines del ejemplo se tiene la cohorte 35-39 años de la Encuesta Mexicana de Fecundidad). Dichas proporciones se estandarizan a un radix de 100 mujeres; después son acumuladas con el fin de obtener las edades del estándar de Coale que corresponde a dichas proporciones acumuladas; esto se lleva a cabo interpolando linealmente el valor de los matrimonios acumulados en el estándar de Coale.

Obtenida la serie de las edades del estándar y la serie de las edades observadas, se grafican, mostrando una correlación lineal entre ellas (ver gráfica 1) por lo que se genera la recta de regresión de la forma:

$$
\mathrm{a}_{\mathrm{s}}=\mathrm{a} \frac{\mathrm{l}}{\mathrm{K}}+\mathrm{d}
$$

donde:

as es la edad estándar

a es la edad observada

Grafica 1

Recta de regresión para la cohorte 35-39 años de la EMF.

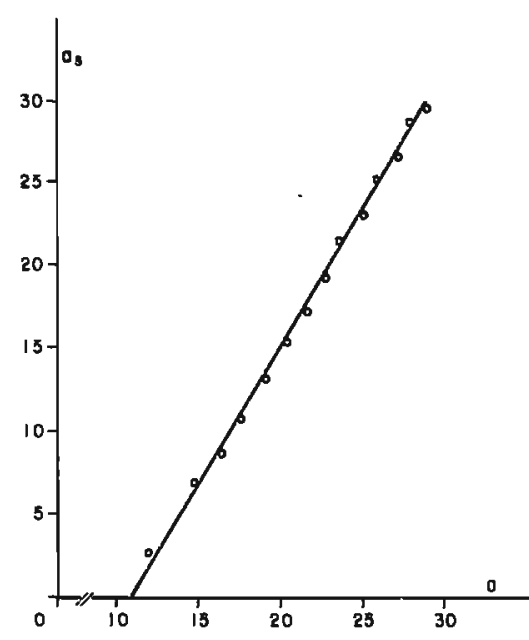


$\frac{1}{\mathrm{~K}}$ es la pendiente de la recta y $\mathrm{K}$ el ritmo al que las mujeres in$\mathrm{K}$ gresan al estado matrimonial.

d es el término independiente de la recta, el cual sirve para determinar el valor de $a_{\mathrm{o}}$ (si $a_{\mathrm{S}}=0$ entonces $\mathrm{a}=\mathrm{a}_{\mathrm{o}}=-\mathrm{dK}$ ).

Con la recta de regresión se obtuvieron las edades as ajustadas (al sustituir en la recta los valores de las edades observadas). Hecho lo anterior, se interpolan linealmente los valores de las edades as ajustadas, teniendo el ajuste de los matrimonios acumulados, los cuales son desacumulados para obtener los matrimonios ocurridos entre las edades a y a+1. En el cuadro 1 se resume el procedimiento antes descrito.

Cuadro 1

México: Ajuste de los primeros matrimonios de la cohorte 35-39 años (EMF) vla el modelo de nupcialidad de coale (por 1000 mujeres).

\begin{tabular}{|c|c|c|c|c|c|c|}
\hline a & $g^{0}(a)$ & $\mathbf{G}^{0}(\mathrm{a})$ & $a_{3}$ & $\hat{a}_{s} \neq 1$ & $\xi(a)$ & $\hat{g}(a)$ \\
\hline 10 & 0 & 0 & 0.00 & 0.00 & 0.00 & 0.00 \\
\hline 11 & 5 & 5 & 1.17 & 0.00 & 0.00 & 0.00 \\
\hline 12 & 11 & 16 & 2.07 & 0.55 & 1.10 & 1.10 \\
\hline 13 & 33 & 49 & 3.37 & 2.37 & 21.53 & 20.43 \\
\hline 14 & 45 & 94 & 4.42 & 4.19 & 82.84 & 61.31 \\
\hline 15 & 78 & 172 & 5.72 & 6.01 & 192.14 & 109.30 \\
\hline 16 & 103 & 275 & 7.13 & 7.83 & 329.63 & 137.49 \\
\hline 17 & 106 & 281 & 8.49 & 9.65 & 469.71 & 140.08 \\
\hline 18 & 111 & 492 & 9.96 & 11.47 & 593.78 & 124.07 \\
\hline 19 & 99 & 591 & 11.42 & 13.29 & 693.71 & 99.93 \\
\hline 20 & 87 & 678 & 12.97 & 15.11 & 771.38 & 77.67 \\
\hline 21 & 78 & 756 & 14.71 & 16.93 & 830.77 & 59.39 \\
\hline 22 & 60 & 816 & 16.42 & 18.75 & 875.16 & 44.39 \\
\hline 23 & 49 & 865 & 18.28 & 20.57 & 908.56 & 33.40 \\
\hline 24 & 30 & 895 & 19.76 & 22.39 & 933.26 & 24.70 \\
\hline 25 & 38 & 933 & 22.37 & 24.21 & 951.08 & 17.82 \\
\hline 26 & 21 & 954 & 24.58 & 26.03 & 963.78 & 12.70 \\
\hline 27 & 24 & 978 & 28.83 & 27.85 & 973.52 & 9.74 \\
\hline 28 & 10 & 988 & 31.64 & 29.67 & 981.42 & 7.90 \\
\hline 29 & 12 & 1000 & 40.00 & 31.49 & 987.57 & 6.15 \\
\hline
\end{tabular}




\subsection{El modelo bilogístico de A. Bocaz ${ }^{3}$}

Si un proceso determinado se inicia a la edad $(\alpha)$ y termina a la edad $(\beta)$, cuando la mujer tenga $(x)$ años de edad habrá recorrido una proporción de tiempo igual a:

$$
\operatorname{Px}=\frac{x-\alpha}{\beta-\alpha}
$$

Si conocemos la proporción de personas que ya han participado en el proceso $(\mathrm{Fx})$, el modelo bilogístico simple presupone una relación lineal entre:

El logito del tiempo recorrido

$$
\operatorname{logit}(\mathrm{Px})=\ln \frac{1}{\mathrm{Px}}-1=\ln \frac{1-\mathrm{Px}}{\mathrm{Px}}
$$

y el logito de la proporción de personas que ya han participado del proceso.

$$
\operatorname{logit}(F x)=\ln \frac{1}{F_{X}}-1=\ln \frac{1-F x}{F_{x}}
$$

en símbolos:

$$
\operatorname{logit}(F y)=b_{1}+b_{2} \operatorname{logit}(P x)
$$

entonces:

$$
\ln \frac{1}{F x}-1=b_{1}+b_{2} \ln \frac{1}{P x}-1
$$

A continuación se da un ejemplo de la aplicación del modelo bilogístico de A. Bo dz tomando la distribución observada de mujeres alguna vez casadas o unidas, según edad al casarse o unirse por primera vez, de la encuesta mexicana de fecundidad (1976).

3 Ver: Bocaz, Albino, "Experiencia de nupcialidad por cohortes resumida por un modelo bilogístico", Notas de Población, año Vll, No. 19, abril, 1979. 
Cuadro 2

Distribución observada de mujeres alguna vez casadas - unidas, según edad al casarse o unirse por primera vez (ECUPV), Cohorte 30-34 años
(EMF, 1976)

\begin{tabular}{lc}
\hline ECUPV & $\begin{array}{c}\text { Proporción de mujeres alguna vez } \\
\text { casadas onidas (por cien) } \\
\text { valores observados }\end{array}$ \\
\hline $10-14$ & 9.1304 \\
$15-17$ & 27.3913 \\
$18-19$ & 18.6957 \\
$20-21$ & 15.5435 \\
$22-23$ & 16.1957 \\
$25-29$ & 10.7609 \\
30 y ms & 2.2826 \\
To t a 1 & 100.0000 \\
\hline
\end{tabular}

En este caso

$\alpha=10$ y $\beta=35$ de donde:

$$
P x=\frac{x-10}{25}
$$

\section{Entonces:}

Cuadro 3

\begin{tabular}{|c|c|c|c|c|c|c|}
\hline $\begin{array}{l}\text { Edad al casarse o } \\
\text { unfrse por la. vez }\end{array}$ & $x$ & $\mathrm{Px}$ & Fx & $F x(x 100)$ & $\log i t P x$ & $\log 1 t F x$ \\
\hline $10-14$ & 15 & .20 & 0.091304 & 9.1304 & 1. 3862944 & 2.3069441 \\
\hline $15-17$ & 18 & .32 & 0.365210 & 36.5210 & 1.7537718 & -2.5527898 \\
\hline $18-19$ & 20 & .40 & 0.552173 & 55.2173 & 0.4054651 & -0.2094581 \\
\hline $20-21$ & 22 & .48 & 0.707608 & 70.7608 & 0.0800427 & -0.8837983 \\
\hline $22-24$ & 25 & .60 & 0.869586 & 86.9566 & -0.4054651 & -1.8971300 \\
\hline $25-29$ & 30 & .80 & 0.977173 & 97.7173 & -1.3869440 & -3.7567606 \\
\hline 30 y mas & 35 & 1.00 & 1.000000 & 100.0000 & - & - \\
\hline
\end{tabular}

Ahora, con el supuesto de una relación de tendencia lineal entre ambos logitos (ver gráfica 2); se determina la recta de regresión siguente:

$\ln \frac{1}{\mathrm{Fx}}-1=-.947272+211542318 \ln \frac{1}{\mathrm{Px}}-1$

$\operatorname{corr}=0.9964262$ 
De (12) se obtienen los valores de $\hat{F}(x)$ siguientes:

Cuadro 4

\begin{tabular}{ccc}
\hline$x$ & logit $\hat{F x}$ & $\hat{F x}$ \\
\hline 15 & 2.0391275 & 0.1151556 \\
18 & 0.6765272 & 0.3370368 \\
20 & -0.0738062 & 0.5184432 \\
22 & -0.7748415 & 0.6845673 \\
25 & -1.8207378 & 0.8606546 \\
30 & -3.9336715 & 0.9808040 \\
\hline
\end{tabular}

Grafica 2

Correlacion entre las transformadas loglsticas de la proporción de mujeres casadas y del tiempo de permanencia en el proceso de nupcialidad. Cohorte de mujeres 30-34 años (EMF, 1976)

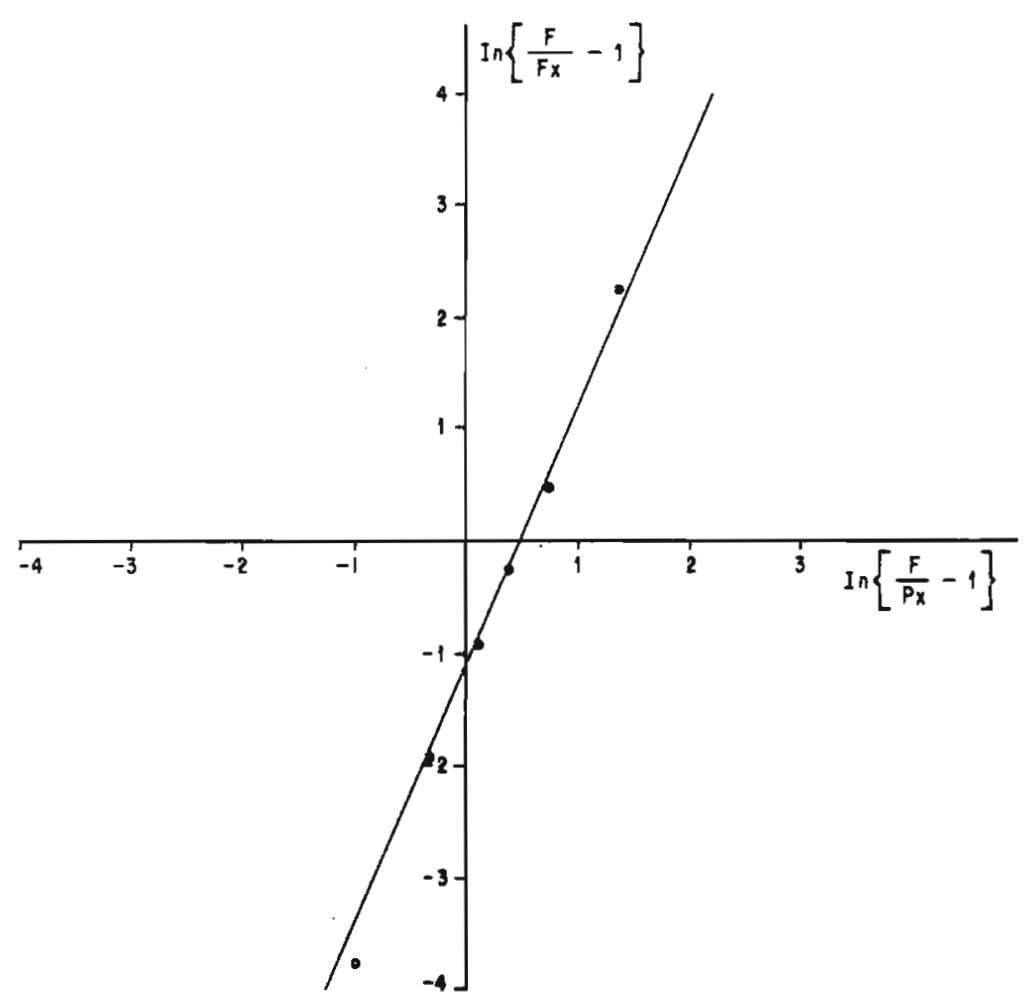


De aquí que la proporción teórica fx, por grupos de edad, estará dada por

$$
\hat{f} x=(\hat{F} x-\hat{F} x-1)
$$

Así:

Cuadro 5

\begin{tabular}{cc}
\hline $\mathrm{x}$ & $\hat{\mathrm{f} x}$ \\
\hline 15 & .1151556 \\
18 & .2218812 \\
20 & .2814064 \\
22 & .1661241 \\
25 & .1760873 \\
30 & .1201494 \\
35 & .0101060 \\
\hline
\end{tabular}

Evaluando la ecuación (12) para las edades exactas: 11, 12, 13, $\ldots, 35$ se obtienen los siguientes valores $\hat{F}(x)$ :

Cuadro 6

\begin{tabular}{llllll}
\hline$x$ & $\hat{F}(x)$ & $x$ & $\hat{F}(x)$ & $x$ & $\hat{F}(x)$ \\
\hline 11 & .0027347 & 20 & .5184432 & 28 & .9517479 \\
12 & .0132018 & 21 & .6053370 & 29 & .9586415 \\
13 & .0340631 & 22 & .6845673 & 30 & .9808040 \\
14 & .0675507 & 23 & .7539335 & 31 & .9892227 \\
15 & .1151556 & 24 & .8125692 & 32 & .9947247 \\
16 & .1771369 & 25 & .8606546 & 33 & .9979921 \\
17 & .2521238 & 26 & .8990524 & 34 & .9995878 \\
18 & .3370368 & 27 & .9289764 & 35 & .9999990 \\
19 & .427664 & & & & \\
\hline
\end{tabular}

Utilizando la ecuación (13), obtenemos los valores $\hat{f}(x)$ para las edades cumplidas: $10,11,12, \ldots, 34$. A continuación se dan los valores, observados y estimados, de la función $\mathrm{f}(\mathrm{x})$ (por cien). 
Cuadro 7

\begin{tabular}{ccccccccc}
\hline$x$ & $\hat{f}(x)$ & $\hat{f}(x)$ & $x$ & $\hat{f}(x)$ & $\hat{f}(x)$ & $x$ & $f(x)$ & $\hat{f}(x)$ \\
\hline 10 & 0.21739 & 0.27347 & 19 & 8.58696 & 9.09768 & 27 & 1.73913 & 2.27150 \\
11 & 0.32609 & 1.04671 & 20 & 9.34783 & 8.68938 & 28 & 1.63043 & 1.68936 \\
12 & 0.97826 & 2.08613 & 21 & 6.19565 & 7.92303 & 29 & 0.97826 & 1.21626 \\
13 & 2.50000 & 3.34876 & 22 & 7.17391 & 6.93662 & 30 & 1.19565 & 0.84187 \\
14 & 5.10870 & 4.76049 & 23 & 5.65217 & 5.86357 & 31 & 0.54348 & 0.55020 \\
15 & 6.63043 & 6.19813 & 24 & 3.36957 & 4.80854 & 32 & 0.32609 & 0.32674 \\
16 & 9.89130 & 7.49869 & 25 & 3.69565 & 3.83978 & 33 & 0.10870 & 0.15957 \\
17 & 10.86957 & 8.49130 & 26 & 2.71739 & 2.99240 & 34 & 0.10870 & 0.04122 \\
18 & 10.10870 & 9.04296 & & & & & & \\
\hline
\end{tabular}

\subsection{Polinomio de fecundidad de W. Brass. *}

E1 Polinomio de fecundidad de Brass se define como:

$\mathrm{f}(a)=\mathrm{c}(\mathrm{x}-\mathrm{S})(\mathrm{S}+33-\mathrm{a})^{2} \quad$ para $\mathrm{S} \leqslant \mathrm{a} \leqslant \mathrm{S}+33$

$f(a)=c(x-S)(b-a)^{2}$

Donde:

c: se relaciona con el nivel de la fecundidad

s: indica la edad en la cual comienza la fecundidad

b: indica la edad en la cual termina la fecundidad $(b=S+33)$

El valor 33 tiene que ver con el intervalo del período reproductivo, es un promedio de numerosas observaciones.

En general se cuenta con las tasas específicas de fecundidad para los grupos quinquenales de edad: $10-14,15-19, \ldots, 40-44$ y $45-49$, las cuales nos dan el comportamiento de dicha función en los puntos $12.5,17.5, \ldots, 42.5$ y 47.5 . Para desagregar dicha información y obtener una completa descripción de la fecundidad por edades individuales se puede utilizar el polinomio de fecundidad de W. Brass.

4 Ver: Brass, W. "Seminario sobre métodos para medir variables demográficas (Fecundidad y mortalidad)". CELADE, Serie DS. No. 9, San José, Costa Rica, 1973, pp. 15-20. 
Las soluciones del polinomio de fecundidad de W. Brass son:

$\mathrm{D}=$ descendencia final $=\frac{\mathrm{c}(\mathrm{b}-\mathrm{S})^{4}}{12}$

12

$\bar{x}=$ edad media a la fecundidad $=\frac{S+2 b}{5}$

$\sigma=$ desviación estándar $=\frac{\mathrm{b}-\mathrm{S}}{5}$

Despejando de las anteriores ecuaciones los valores de S, b y c:

$$
\begin{aligned}
& \mathrm{S}=\overline{\mathrm{x}}-25 \\
& \mathrm{~b}=\overline{\mathrm{x}}+35 \\
& \mathrm{Km}=\frac{12 \mathrm{D}}{(\mathrm{b}-\mathrm{S})^{4}}
\end{aligned}
$$

Ejemplo de la aplicación del polinomio de W. Brass: Para la región 3 , que comprende los estados de Tamaulipas y Veracruz, se obtiene para 1970:

\begin{tabular}{ccccc}
\multicolumn{5}{c}{ Cuadro 8} \\
\hline $\begin{array}{c}\text { Grupos } \\
\text { de } \\
\text { edad }\end{array}$ & $\begin{array}{c}\text { Promedio de } \\
\text { nacimientos } \\
1969-71 \text { If }\end{array}$ & $\begin{array}{c}\text { Total de } \\
\text { mujeres al } \\
30.06 .702 /\end{array}$ & $\begin{array}{c}\text { Tasas especificas } \\
\text { de } \\
\text { fecundidad } f(a)\end{array}$ & $a$ \\
\hline $12-14$ & 1198 & 200329 & 0.005980 & 13.5 \\
$15-19$ & 21306 & 280781 & 0.075881 & 17.5 \\
$20-24$ & 53031 & 234961 & 0.225701 & 22.5 \\
$25-29$ & 48797 & 193137 & 0.252655 & 27.5 \\
$30-34$ & 33737 & 149714 & 0.225343 & 32.5 \\
$35-39$ & 24073 & 147849 & 0.162822 & 37.5 \\
$40-49$ & 15683 & 196067 & 0.079988 & 43.3 \\
\hline
\end{tabular}

Fuente: 1/ Anuario Estadistico, México, 1972.

2/ IX Censo General de Poblacion, México, 1972.

En este caso:

$$
\mathrm{D}=5 \underset{a=13.5}{\sum_{a} .3} \mathrm{f}(a)=5(1.028370)=5.14185
$$




$$
\begin{aligned}
& \overline{\mathrm{x}}=\frac{\sum_{a=13.5}^{43.3} \mathrm{f}(a)}{43.3} \\
& a=13.5 \mathrm{f}(a) \\
& a=\sqrt{\sum_{13.5}^{43.3} a^{2} \mathrm{f}^{\prime}(a)-\overline{\mathrm{x}}^{2}}=6.826578
\end{aligned}
$$

Por lo tanto:

$$
\begin{aligned}
& \mathrm{S}=\overline{\mathrm{x}}-2 \sigma=15.838044 \\
& \mathrm{~b}=\overline{\mathrm{x}}+3 \sigma=49.970934 \\
& \mathrm{c}=\frac{12 \mathrm{D}}{(\mathrm{b}-\mathrm{S})^{4}}=0.0000454578
\end{aligned}
$$

Finalmente se obtiene la función de fecundidad:

$$
f(a)=0.0000454578(a-15.838044)(49.970934-a)^{2}
$$

Obsérvese que se puede evaluar la función de fecundidad $\mathrm{f}(a)$ para toda $a$ mayor a 15.838044 y menor a 49.970934 , desagregando de grupos quinquenales a edades individuales la experiencia en cuanto a la fecundidad de dicha región, lo cual se da en el Cuadro 9.

\subsection{Modelo de fecundidad de A. J. Coale y J. Trussell ${ }^{5}$}

Este modelo se basa en el modelo estándar de nupcialidad de A. J. Coale; parte de la función G(a) (función acumulada de los primeros matrimonios ocurridos hasta la edad a) y de la función r(a) que representa la proporción de mujeres casadas con hijos nacidos vivos en la edad a (fecundidad de las casadas).

La forma de la función $\mathrm{f}(a)$ es:

$$
f(a)=G(a) r(a)
$$

5 Ver: Coale, A.J. y Trussell, T.J.: "Model Fertility Schedules: Variations in the age structure of childbearing in human populations". Population Index. 40 (2). pp. 185-258. 1974. 
Cuadro 9

Region 3. Tamaulipas y Veracruz. Tasas especificas de fecundidad, obtenidas a partir de la función de fecundidad de $\mathrm{h}$. Brass.

\begin{tabular}{cccccc}
\hline$x$ & $f(x)$ & $x$ & $f(x)$ & $x$ & $f(x)$ \\
\hline 16.5 & 0.03371 & 28.5 & 0.26535 & 40.5 & 0.10056 \\
17.5 & 0.07966 & 29.5 & 0.26025 & 41.5 & 0.08371 \\
18.5 & 0.11985 & 30.5 & 0.25268 & 42.5 & 0.06765 \\
19.5 & 0.15456 & 31.5 & 0.24290 & 43.5 & 0.05265 \\
20.5 & 0.18406 & 32.5 & 0.23119 & 44.5 & 0.03900 \\
21.5 & 0.20863 & 33.5 & 0.21781 & 45.5 & 0.02695 \\
22.5 & 0.22854 & 34.5 & 0.20305 & 46.5 & 0.01679 \\
23.5 & 0.24405 & 35.5 & 0.18717 & 47.5 & 0.00878 \\
24.5 & 0.25545 & 36.5 & 0.17044 & 48.5 & 0.00321 \\
25.5 & 0.26301 & 37.5 & 0.15315 & 49.5 & 0.00034 \\
26.5 & 0.26700 & 38.5 & 0.13555 & & \\
27.5 & 0.26768 & 39.5 & 0.11793 & & \\
\hline
\end{tabular}

siendo: $r(a)=n(a) \cdot M \cdot \exp [m \cdot v(a)]$

donde:

$\mathrm{n}$ (a) representa la fecundidad natural a edad a (obtenida a partir de la experiencia de 43 países).

M es un factor de escala (como el valor $\mathrm{C}$ del modelo estándar de nupcialidad de Coale).

v(a) expresa la tendencia de las mujeres en edad avanzada al uso de anticonceptivos y aborto.

m denota el grado de control de los niveles de fecundidad.

Los valores de n(a) y v(a) para los grupos de edad $20-24,25-29, \ldots$, 40-44 y 45-49, son los siguientes:

Cuadro 10

\begin{tabular}{ccccccc}
\hline Edad & $20-24$ & $25-29$ & $30-34$ & $35-39$ & $40-44$ & $45-49$ \\
\hline$\Omega(a)$ & 0.460 & 0.431 & 0.396 & 0.321 & 0.167 & 0.024 \\
$v(a)$ & 0.000 & -0.316 & -0.814 & -1.048 & -0.424 & -1.667 \\
\hline
\end{tabular}


El modelo de fecundidad de Coale y Trussell se emplea estandarizado a 1.0 la suma de las tasas específicas de fecundidad. Coale obtiene los valores de las tasas específicas de fecundidad desagregadas en edades individuales para los siguientes parámetros:

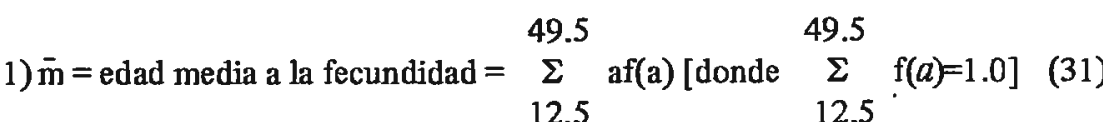

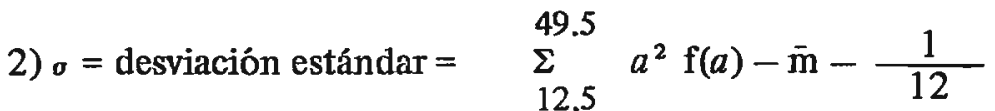

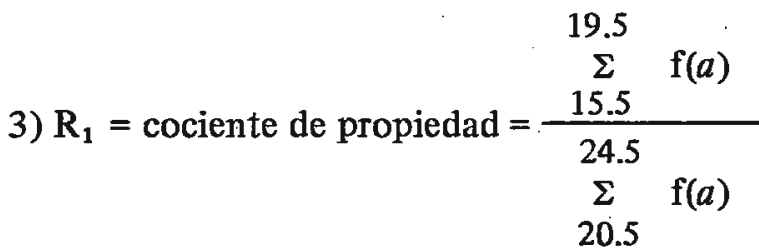

4) $\mathrm{MED}=$ el valor de â tal que $\stackrel{1}{\Sigma}_{12.5} \mathrm{f}(a)=0.5$

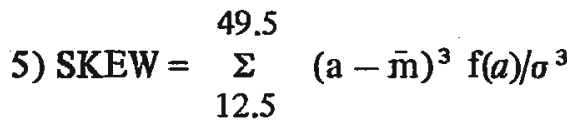

6) PAR $1 \times \frac{\text { Paridad }(15-19)}{\text { Paridad }(20-24)}$

7) PAR $2=\frac{\text { Paridad }(20-24)}{\text { Paridad }(25-29)}$

8) $a_{o}=$ edad inicial al matrimonio en la función de nupcialidad

9) $\mathrm{K}=$ factor de escala de la función de nupcialidad.

10) $\mathrm{m}=$ grado de control de la fecundidad. 
Con diferentes combinaciones de los anteriores parámetros los autores crearon tablas que muestran la distribución de las tasas específicas de fecundidad, ${ }^{6}$ las cuales se utilizan como estándar para obtener la distribución de dichas tasas para el caso particular que se tenga.

La forma en que se deben utilizar las tablas antes citadas es la siguente:

a) Se obtienen los valores de los parámetros $\overline{\mathrm{m}}, \sigma$ y $\mathrm{R}_{1}$.

b) Se buscan los valores de $R_{1}$ en las tablas que cubra el valor observado de dicho parámetro y a los parámetros $\overline{\mathrm{m}}$ y o como se muestra en la siguiente gráfica.

Grafica 3

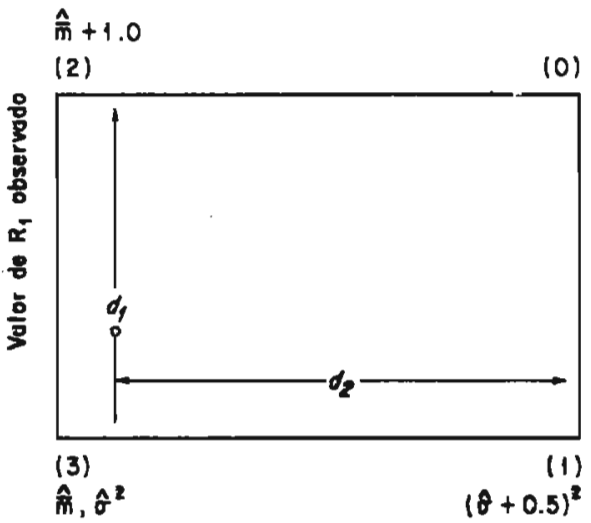

donde:

$$
\begin{aligned}
& \mathrm{d}_{1}=(\hat{\overline{\mathrm{m}}}+1.0)-\overline{\mathrm{m}} \\
& \mathrm{d}_{2}=\frac{(\hat{\sigma}+0.5)-\sigma}{(\hat{\sigma}+0.5)-\sigma^{2}}
\end{aligned}
$$

(0) es el punto $P_{0}=\left[(\hat{\sigma}+0.5)^{2},(\hat{\overline{\mathrm{m}}}+1.0)\right]$

(1) es el punto $P_{1}=\left[(\hat{\sigma}+0.5)^{2}, \hat{\bar{m}}\right]$

6 Coaje, A.J. y Trussell T.J., op. cit., pp. 205-257. 
(2) es el punto $P_{2}=\left[\hat{\sigma}^{2},(\hat{\overline{\mathrm{m}}}+1.0)\right]$

E1 Punto (3) debe ser tal que el valor que se tiene para él es el menor de los tres puntos anteriores, siendo el punto que se elimina.

c) De cada punto $\left(\mathrm{P}_{0}, \mathrm{P}_{1}\right.$ y $\left.\mathrm{P}_{2}\right)$ se toman los valores de los parámetros $\mathrm{a}_{\mathrm{o}}, \mathrm{K}$ y $\mathrm{m}$ que se dan en las tablas correspondientes.

Así se tiene:

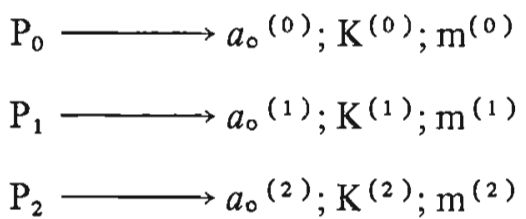

d) Se calculan los valores de los parámetros ponderadores, $W_{0}, W_{1}$ y $\mathrm{W}_{2}$, como sigue:

$\mathrm{W}_{1}=\mathrm{d}_{1}$

$\dot{\mathrm{W}}_{2}=\mathrm{d}_{2}^{*}=\mathrm{d}_{2}+\frac{\mathrm{d}_{1}\left(1-\mathrm{d}_{1}\right)}{\sigma+0.25}$

$\mathrm{W}_{0}=1-\mathrm{W}_{1}-\mathrm{W}_{2}$

e) Se obtienen los valores de los parámetros $a_{\circ}, \mathrm{K}$ y $\mathrm{m}$ para la fecundidad observada.

$\hat{a}=\mathrm{W}_{0} a_{0}{ }^{(0)}+\mathrm{W}_{1} a_{0}^{(1)}+\mathrm{W}_{2} a_{0}{ }^{(2)}$

$\hat{\mathrm{K}}=\mathrm{W}_{0} \mathrm{~K}^{(0)}+\mathrm{W}_{1} \mathrm{~K}^{(1)}+\mathrm{W}_{2} \mathrm{~K}^{(2)}$.

$\hat{\mathrm{m}}=\mathrm{W}_{0} \mathrm{~m}^{(0)}+\mathrm{W}_{1} \mathrm{~m}^{(1)}+\mathrm{W}_{2} \mathrm{~m}^{(2)}$

f) Se evalúa la función $\mathrm{f}(\mathrm{a})=\mathrm{G}(\mathrm{a}) \mathrm{n}(\mathrm{a}) e^{\mathrm{m}} \mathrm{v}(\mathrm{a}),{ }^{7}$ obteniendo los valores, corregidos y desagregados por edad, de la distribución de las tasas específicas de fecundidad correspondientes a la experiencia observada.

7 Empleando el programa de computación que aparece en las páginas 202 y 203 del artículo de Coale y Trussell antes citado. 
Ejemplo de la aplicación del modelo de fecundidad de A. J. Coale y J. Trussell.

Tomando también la región 3, que comprende los estados de Tamaulipas y Veracruz, se tienen las siguientes tasas específicas de fecundidad.

Cuadro 11

\begin{tabular}{cccc}
\hline $\begin{array}{c}\text { Grupos de } \\
\text { edad }\end{array}$ & $\mathrm{a}$ & $\mathrm{f}(\mathrm{a})$ & $f^{\mathrm{s}(a)}$ \\
\hline $12-14$ & 13.5 & 0.005980 & 0.005815 \\
$15-19$ & 17.5 & 0.075881 & 0.073788 \\
$20-24$ & 22.5 & 0.225701 & 0.219475 \\
$25-29$ & 27.5 & 0.252655 & 0.245685 \\
$30-34$ & 32.5 & 0.225343 & 0.219126 \\
$35-39$ & 37.5 & 0.162822 & 0.158330 \\
$40-49$ & 43.3 & 0.079988 & 0.077781 \\
\hline
\end{tabular}

De Donde:

$$
\overline{\mathrm{m}}=\underset{13.5}{\sum^{43.3}} \text { afs }(\mathrm{a})=29.4912
$$

$$
\sigma=\sqrt{\sum_{13.5}^{43.3} a^{2} f^{s}(a)-\bar{m}^{2}-\frac{5^{2}}{12}}=6.9775
$$

$$
R=\frac{0.073788}{0.219475}=0.336202
$$

Buscando los valores de $R_{1}$ en las tablas de Coale y Trussell, tales que cubran los valores de $R_{1}, \bar{m}$ y $o$ observados:

Cuadro 12

\begin{tabular}{c|cc}
\hline $\bar{x}$ & 6.5 & 7.0 \\
\hline 29 & 0.2860 & 0.3370 \\
& & \\
30 & 0.2457 & 0.3319 \\
\hline
\end{tabular}


En este caso:

$$
\begin{aligned}
& \mathrm{P}_{0}=(7,29) ; \mathrm{P}_{1}=(7,30) \text { y } \mathrm{P}_{2}=(6.5,29) \\
& a_{0}{ }^{(0)}=15.39 ; \mathrm{K}^{(0)}=0.300 ; \mathrm{m}^{(0)}=0.282 \\
& a_{0}^{(1)}=12.74 ; \mathrm{K}^{(1)}=0.750 ; \mathrm{m}^{(1)}=0.230 \\
& a_{0}^{(2)}=12.56 ; \mathrm{K}^{(2)}=1.000 ; \mathrm{m}^{(2)}=0.899 \\
& \mathrm{~W}_{1}=\mathrm{d}_{1}=29.4912-29=0.4912 \\
& \mathrm{~d}_{2}=\frac{7^{2}-(6.9775)^{2}}{7^{2}-(6.5)^{2}}=0.04659 \\
& \mathrm{~W}_{2}=\mathrm{d}_{2}^{*}=\mathrm{d}_{2}+\frac{\mathrm{d}_{1}\left(1-\mathrm{d}_{1}\right)}{\sigma+0.25}=0.083618 \\
& \mathrm{~W}_{0}=1-\mathrm{W}_{1}-\mathrm{W}_{2}=0.425182
\end{aligned}
$$

\begin{tabular}{|c|c|c|c|c|c|c|c|}
\hline \multirow{2}{*}{$\bar{x}$} & \multicolumn{6}{|c|}{ Cuadro 13} & \multirow[b]{2}{*}{$f(x)$} \\
\hline & $x)$ & $\mathrm{x}$ & $f(x)$ & $\mathbf{x}$ & $f(x)$ & $\mathbf{x}$ & \\
\hline ? & 101 & 23 & 0.2560 & 33 & 0.20776 & 43 & 0.05470 \\
\hline 1 . & U. $) 7$ & 24 & 0.2602 & 34 & 0.19646 & 44 & 3.03697 \\
\hline 15 & $0 . \quad$ י & 25 & $0.26^{\circ} .4$ & 35 & 0.18489 & 45 & .02294 \\
\hline 16 & $U_{<} 8 G$ & 26 & 0.2636 & 36 & 0.17261 & 46 & .01438 \\
\hline 17 & 0.0637 & 7 & 0.259 .1 & 37 & .16021 & 47 & $\therefore .00855$ \\
\hline 18 & 0.11116 & 2 & 0.2539 & 38 & .14725 & $4 \varepsilon$ & 00445 \\
\hline 19 & 0.15695 & $2 !$ & 0.247 & 39 & $1.13 i 6$ & 4 & 00121 \\
\hline 20 & 0.19471 & 3. & $12 x+3$ & 40 & 1.1132 & & \\
\hline 21 & 0.22271 & 31 & $0 . .837$ & 41 & $.09 \quad 79$ & & \\
\hline 22 & 0.24187 & 32 & $\therefore ? 1829$ & 42 & $0 . \quad 4=$ & & \\
\hline
\end{tabular}

Finalmente:

$$
\begin{aligned}
& \hat{a}_{0}=\mathrm{W}_{0} a_{0}^{(0)}+\mathrm{W}_{1} a_{0}^{(1)}+\mathrm{W}_{2} a_{0}^{(2)}=13.8517 \\
& \hat{\mathrm{K}}=\mathrm{W}_{0} \mathrm{~K}^{(0)}+\mathrm{W}_{1} \mathrm{~K}^{(1)}+\mathrm{W}_{2} \mathrm{~K}^{(2)}=0.5796 \\
& \hat{\mathrm{n}}=\mathrm{I}_{0} \mathrm{~m}^{(0)}+\mathrm{W}_{1} \mathrm{~m}^{(1)}+\mathrm{W}_{2} \mathrm{~m}^{(2)}=0.3080
\end{aligned}
$$

Con $\hat{z}_{\circ}, \hat{\mathrm{K}}$ y $\mathrm{m}$ y usando el programa de computación se o: tiene la zuien : estructura por edad de la fecundidad: 


\subsection{La función de Makeham}

Uno de los esfuerzos para dar una ley matemática que describiera la mortalidad experimentada por una población dada, fue el hecho por Gompertz. La ley que desarrolló (Ley de Gompertz) supone que la resistencia del hombre a la muerte decrece a una tasa proporcional a sí misma, y que las causas de muerte se agrupan en: a) aquellas independientes de la edad y b) aquellas en las que el organismo ofrece una resistencia que se va perdiendo con el tiempo.

Gompertz, en su ley, sólo considera las causas de muerte dependientes de la edad y Makeham, basado en la ley de Gompertz, integra las causas independientes de la edad para generar su ley.

Si se denota como Mx (tasa instantánea de mortalidad) a la susceptibilidad del hombre a la muerte, y al recíproco de ella, $\frac{1}{\mathrm{Mx}}$, como la resistencia del hombre a la muerte, entonces, el supuesto de la ley de Gompertz se puede expresar de la siguiente manera:

$$
\frac{d}{d x}(1 / M x)=-h(1 / M x)
$$

donde $\mathrm{h}$ es la tasa a la cual decrece la resistencia del hombre a la muerte.

Desarrollando (66):

$$
\begin{aligned}
& \int \frac{\mathrm{d}(1 / \mathrm{Mx})}{1 / \mathrm{Mx}} \mathrm{dx}=-\mathrm{h} \int \mathrm{dx} \\
& \ln (1 / \mathrm{Mx})+\ln B=-\mathrm{hx} \\
& \ln \frac{\mathrm{B}}{\mathrm{Mx}}=-\mathrm{hx} \\
& \frac{\mathrm{B}}{\mathrm{Mx}}=-\mathrm{hx} \\
& \mathrm{i}=\quad 3 \mathrm{H}^{\mathrm{hx}}
\end{aligned}
$$

Se den sta a $e^{\mathrm{h}}$ como $\mathrm{C}$, entonces (71) se expresa como:

$$
\mathrm{Mx}=\mathrm{BC}^{\mathrm{x}}
$$


Por otro lado, la tasa instantánea de mortalidad Mx se define como:

$$
\begin{aligned}
M x & =\lim _{h \rightarrow 0} \frac{\ell(x)-\ell(x+h)}{h \ell(x)} \\
& =\frac{-1}{\ell(x)} \lim _{h \rightarrow 0} \frac{\ell(x+h)-\ell(x)}{h} \\
& =-\frac{1}{\ell(x)} \frac{1}{d x} \ell(x) \\
& =-\frac{d}{d x} \ln \ell(x)
\end{aligned}
$$

De (76):

$$
\begin{aligned}
\int_{0}^{x} M y d y & =-\int_{0}^{x} \frac{d}{d y} \ln \ell(y) d y \\
& =-\ln \ell(y) \int_{0}^{x} \\
& =-[\ln \ell(x)-\ln \ell(0)] \\
& =-\ln \frac{\ell(x)}{\ell(0)}
\end{aligned}
$$

Por lo tanto:

$$
\begin{aligned}
& \ln \frac{\ell(x)}{\ell \cdot(0)}=-\int_{0}^{x} M y d y \\
& \frac{\ell(x)}{\ell(0)}=e^{--0} M y d y \\
& \ell(x)=\ell(0) e^{-\int_{\overline{0}}^{x} M y d y}
\end{aligned}
$$


Makeham, al combinar las dos causas de muerte supuestas por Gompertz, considera la expresión (7) como:

$$
\mathrm{Mx}=\mathrm{A}+\mathrm{BC}^{\mathrm{x}}
$$

En donde el parámetro A está asociado al efecto de las causas de muerte independientes de la edad.

Desarrollando (84):

$$
\begin{aligned}
\int_{0}^{\mathrm{x} y d y} & =\int_{0}^{\mathrm{x}}\left(\mathrm{a}+\mathrm{BC} C^{\mathrm{y}}\right) \mathrm{dy} \\
& =\int_{0}^{\mathrm{x}} \mathrm{Ady}+\int_{0}^{\mathrm{x}} \mathrm{BC}^{\mathrm{y}} \mathrm{dy} \\
& =\mathrm{Ax}+\frac{\mathrm{BC}}{\operatorname{lnC}}-\frac{\mathrm{B}}{\operatorname{lnC}} \\
-\int_{0}^{\mathrm{x}} \mathrm{Mydy} & =-\mathrm{Ax}-\frac{\mathrm{B}}{\operatorname{lnC}}\left(C^{\mathrm{x}}-1\right)
\end{aligned}
$$
Se denota a $-\mathrm{A}$ como $\ln S$ y a $\frac{-\mathrm{B}}{\operatorname{lnC}}$ como $\operatorname{lng}$, entonces (88) se
expresa como:

$$
\begin{aligned}
-\int_{0}^{x} M y d y= & x \ln S+\left(C^{x}-1\right) \operatorname{lng} \\
& \ln S^{x}+\operatorname{lng}\left(C^{x}-1\right)
\end{aligned}
$$

Sustituyendo (90 en (83):

$$
\begin{aligned}
& \ell(x)=\operatorname{loS}^{x} g\left(C^{x}-1\right) \\
& =\frac{\ell O}{g} S^{x} g C^{x}
\end{aligned}
$$


Se denota a $\frac{\ell 0}{g}$ como K, entonces (92) se expresa como:

$$
\ell(\mathrm{x})=\mathrm{KS}^{\mathrm{x}} \mathrm{g}^{\mathrm{x}}
$$

La expresión (93) es la comúnmente llamada ley de Makeham, siendo la siguiente expresión la llamada función de Makeham:

$$
Y(x)=K a^{x} b^{d^{x}}
$$

Para determinar los valores de los parámetros $\mathrm{K}$, a, b y d se usa el método de los grupos no superpuestos ${ }^{8}$ el cual consiste en separar los datos observados $[\mathrm{Y}(\mathrm{x})]$ en cuatro grupos de observaciones sucesivas, con un número igual de valores por grupo. Es decir:

Primer grupo:

$\mathrm{x}: \begin{array}{llll}1 & 2 & 3 & \ldots(\mathrm{m}-1)\end{array}$

$\mathrm{Yx}: \mathrm{Y}_{0} \mathrm{Y}_{1} \mathrm{Y}_{2} \ldots \mathrm{Ym}_{\mathrm{M}}-1$

Segundo grupo:

$\mathrm{x}: \mathrm{m}(\mathrm{m}+1)(\mathrm{m}+2) \cdot \cdot \cdot(2 \mathrm{~m}-1)$

$Y x: Y m Y m+1 Y m+2$. . . $Y_{2 m}-1$

Tercer grupo:

$x: 2 m(2 m+1)(2 m+2) \cdot \cdot \cdot(3 m-1)$

$Y x: Y_{2 m} Y_{2 m}+1 Y_{2 m}+2 \ldots Y_{3 m}-1$

Cuarto grupo:

$x: 3 m(3 m+1)(3 m+2) \ldots \cdot(4 n-1)$

$Y x: Y_{3 m} Y_{3 m}+1 Y_{3 m}+2 \ldots Y_{4,}-1$

Ca culando los logari nos decimai ss para cada una de las observaciones:

$\log \mathrm{Y}(\mathrm{i})=\log \mathrm{K}+\mathrm{i} \log \mathrm{a} \cdot \mathrm{d}^{\mathrm{i}} \log \mathrm{b}$ para toda $\mathrm{i}=0,1,2, \ldots,(4 \mathrm{~m}-1)$

P h. : Albin Bt 'a :, "El uso de la ley de Makcham cono función demográfica", Notas ac Pot ac:o:l, CI 'ADE uñ II, Vol. 6, diciembre, 1974, f'g 41. 
y sumando dichos logoritmos para cada grupo, se obtiene (sean $\mathrm{S}_{1}$, $S_{2}$ y $S_{3}$ los resultados de dichas sumas):

$$
\begin{aligned}
& \mathrm{S}_{0}=\mathrm{m} \log \mathrm{K}+\frac{\mathrm{m}(\mathrm{m}-1)}{2} \log \mathrm{a}+\frac{\mathrm{d}^{\mathrm{m}}-1}{\mathrm{~d}-1} \log \mathrm{b} \\
& \mathrm{S}_{1}=\mathrm{m} \log \mathrm{k}+\mathrm{m}^{2}+\frac{\mathrm{m}(\mathrm{m}-1)}{2} \log \mathrm{a}+\mathrm{d}^{\mathrm{m}} \frac{\mathrm{d}^{\mathrm{m}}-1}{\mathrm{~d}-1} \log \mathrm{b}(105) \\
& \mathrm{S}_{2}=\mathrm{m} \log \mathrm{K}+2 \mathrm{~m}^{2}+\frac{\mathrm{m}(\mathrm{m}-1)}{2} \log \mathrm{a}+\mathrm{d}^{2} \mathrm{~m} \frac{\mathrm{d}^{\mathrm{m}}-1}{\mathrm{~d}-1} \operatorname{logh} \\
& \mathrm{S}_{3}=\mathrm{m} \log \mathrm{K}+3 \mathrm{~m}^{2}+\frac{\mathrm{m}(\mathrm{m}-1)}{2} \log \mathrm{a}+\mathrm{d}^{3 \mathrm{~m}} \frac{\mathrm{d}^{\mathrm{m}}-1}{\mathrm{~d}-1} \log \mathrm{b}
\end{aligned}
$$

Calculando las primeras y segundas diferencias de las anteriores sumas, se tiene:

$$
\begin{aligned}
& \Delta \mathrm{S}_{0}=\mathrm{S}_{1}-\mathrm{S}_{0} \\
& =m^{2} \log a+\frac{\left(d^{m}-1\right)^{2}}{d-1}-\log b \\
& \Delta \mathrm{S}_{1}=\mathrm{S}_{2}-\mathrm{S}_{1} \\
& =m^{2} \log a+d^{m} \frac{\left(d^{m}-1\right)^{2}}{d-1} \log b \\
& \Delta \mathrm{S}_{2}=\mathrm{S}_{3}-\mathrm{S}_{2} \\
& =m^{2} \log a+d^{2} n \frac{\left(d^{m}-1\right)^{2}}{d-1} \log b \\
& \int^{2} S_{0}=\Delta \dot{i}-\Delta S_{0} \\
& =-\frac{\left(d^{m}-1\right)^{3}}{d-1} \log b \\
& \Delta^{2} S_{1}=\Delta S_{2}-\Delta S_{2}-\Delta S_{1} \\
& =d^{m} \frac{\left(d^{m}-1\right)^{3}}{d-1} \log b
\end{aligned}
$$


Dividiendo (117) entre (115):

$\mathrm{d}=\frac{\Delta^{2} \mathrm{~S}_{1}}{\Delta^{2} \mathrm{~S}_{0}}$

$\mathrm{d}=\left[\frac{\Delta^{2} \mathrm{~S}_{1}}{\Delta^{2} \mathrm{~S}_{1}}\right]^{1 / \mathrm{m}}$

De (41):

$$
\begin{aligned}
\log b & =\frac{\mathrm{d}-1}{\left(\mathrm{~d}^{\mathrm{m}}-1\right)^{3}} \Delta^{2} S_{0} \\
\mathrm{~b} & =\operatorname{anti\ell og}\left[\frac{\mathrm{d}-1}{\left(\mathrm{~d}^{\mathrm{m}}-1\right)^{3}} \Delta^{2} \mathrm{~S}_{0}\right]
\end{aligned}
$$

De (35) y (39):

$$
\begin{aligned}
\log \mathrm{a} & =\frac{1}{\mathrm{~m}^{2}} \Delta \mathrm{S}_{0}-\frac{\Delta^{2} \mathrm{~S}_{0}}{\mathrm{~d}^{\mathrm{m}}-1} \\
\mathrm{a} & =\operatorname{anti\ell \circ g}\left[\frac{1}{\mathrm{~m}^{2}} \Delta \mathrm{S}_{0} \frac{\Delta^{2} \mathrm{~S}_{0}}{\mathrm{~d}^{\mathrm{m}}-1}\right]
\end{aligned}
$$

Finalmente, el parámetro $\mathrm{K}$ se estima a partir de la condición de mínimos cuadrados:

$$
Q=\sum_{0}^{4 m-1}\left(Y x-K a^{X} b^{d x}\right)^{2}=0
$$

Sea $V x=a^{x} b^{d x}$, entonces (124) se expresa como:

$$
\begin{aligned}
& \stackrel{4 m-1}{\sum_{0}}(\mathrm{YX}-\mathrm{XVx})^{2}=0 \\
= & \left(\mathrm{Y}_{\mathrm{X}}^{2}-2 \mathrm{KYX} \mathrm{Vx}+\mathrm{K}^{2} \mathrm{Vx}^{2}\right) \\
= & \left(\mathrm{Y}_{\mathrm{X}}^{2}-2 \mathrm{Y}_{\mathrm{X}}^{2}+\mathrm{K}^{2} \mathrm{~V}_{\mathrm{X}}^{2}\right) \\
= & \left(\mathrm{K}^{2} \mathrm{~V}_{\mathrm{X}}^{2}-\mathrm{Y}_{\mathrm{X}}^{2}\right)
\end{aligned}
$$


Entonces:

$$
\begin{aligned}
& \begin{array}{c}
{ }^{4 \mathrm{~m}-1} \\
0
\end{array} \mathrm{~K}^{2} \mathrm{~V}_{\mathrm{X}}^{2}=\begin{array}{c}
\sum^{4 \mathrm{~m}-1} \\
0
\end{array} \mathrm{Y}_{\mathrm{X}}^{2}
\end{aligned}
$$

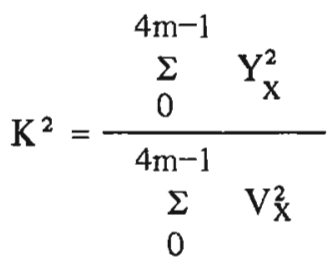

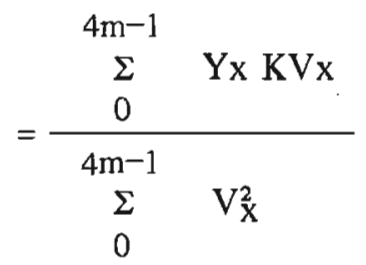

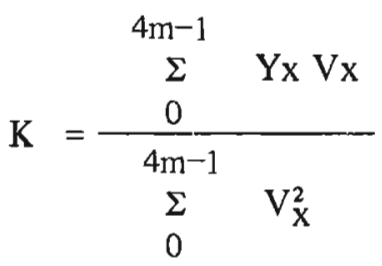

Ejemplos de la aplicación de la función de Makeham.

Tomando la serie $\ell(x)$ de la tabla de mortalidad para los hombres en 1975 a nivel nacional. Se mostrará que la función de Makeham sirve para describir y desagregar la serie indicada. La información empleada aparece en el cuadro 14.

Cabe señalar que para la serie $\mathrm{q}(\mathrm{x})$ sólo para los valores de ella en los grupos de edades 10-14, 15-19, ..., 60-64 y 65-69 se tiene una buena descripción del fenómeno.

Obtenida la serie de valores $\hat{Y} \mathrm{x}$, los cuales representan los valores acumulados de la serie $\mathrm{q}(\mathrm{x})$ estimada, se desacumulan dichos valores obteniendo los valores estimados de la serie $\mathrm{q}(\mathrm{x})$ (ver Cuadro 15).

Para la serie $\ell(x)$ se tiene que la función de Makeham describe el comportamiento de ella desde la edad cero hasta la edad 70 .

A continuación se resumen los pasos que se siguieron para obtener la distribución de la serie $\ell(x)$ (hombres, nivel nacional, 1975), vía la función de Makeham. 
Cuadro 14

México: Valores $q(x)$ y $\ell(x)$, Hombres 1975 .

\begin{tabular}{|c|c|c|c|}
\hline Edad & $q(x)$ & el & $(x)$ \\
\hline 0 & 0.05178 & 100 & 000 \\
\hline $1-4$ & 0.01672 & 94 & 822 \\
\hline $5-9$ & 0.00593 & 93 & 236 \\
\hline $10-14$ & 0.00489 & 92 & 683 \\
\hline $15-19$ & 0.00950 & 92 & 230 \\
\hline $20-24$ & 0.01464 & 91 & 354 \\
\hline $25-29$ & 0.01784 & 90 & 016 \\
\hline $30-34$ & 0.01946 & 88 & 410 \\
\hline $35-39$ & 0.02961 & 86 & 690 \\
\hline $40-44$ & 0.03358 & 84 & 123 \\
\hline $45-49$ & 0.04487 & 81 & 299 \\
\hline $50-54$ & 0.05943 & 77 & 651 \\
\hline $55-59$ & 0.07766 & 73 & 036 \\
\hline $60-64$ & 0.11432 & 67 & 364 \\
\hline $65-69$ & 0.14986 & 59 & 663 \\
\hline $70-74$ & 0.21898 & 50 & 722 \\
\hline $75-79$ & 0.31455 & 39 & 615 \\
\hline $80 y+$ & 1.00000 & 27 & 154 \\
\hline
\end{tabular}

Fuente: S.S.A. Subsecretaria de Planeacion, "Tablas abreviadas por estados y para ocho regiones de Mexico, 1975".

Cuadro 15

Mêxico: Distribución de la función $q(x)$ usando la función de Makeham. Hombres, 1975.

\begin{tabular}{ccc}
\hline $\begin{array}{c}\text { Grupos de } \\
\text { edad }\end{array}$ & $\begin{array}{c}q(x) \\
\text { observadas }\end{array}$ & $\begin{array}{c}q(x) \\
\text { estimadas }\end{array}$ \\
\hline $10-14$ & .00489 & .00477 \\
$15-19$ & .00950 & .01005 \\
$20-24$ & .01464 & .01439 \\
$25-29$ & .01784 & .01754 \\
$30-34$ & .01946 & .02128 \\
$35-39$ & .02961 & .02672 \\
$40-44$ & .03358 & .03461 \\
$45-49$ & .04487 & .04563 \\
$50-54$ & .05943 & .06074 \\
$55-59$ & .07766 & .08121 \\
$60-64$ & .11432 & .10882 \\
$65-69$ & .14986 & .14595 \\
\hline
\end{tabular}


Cuadro 16

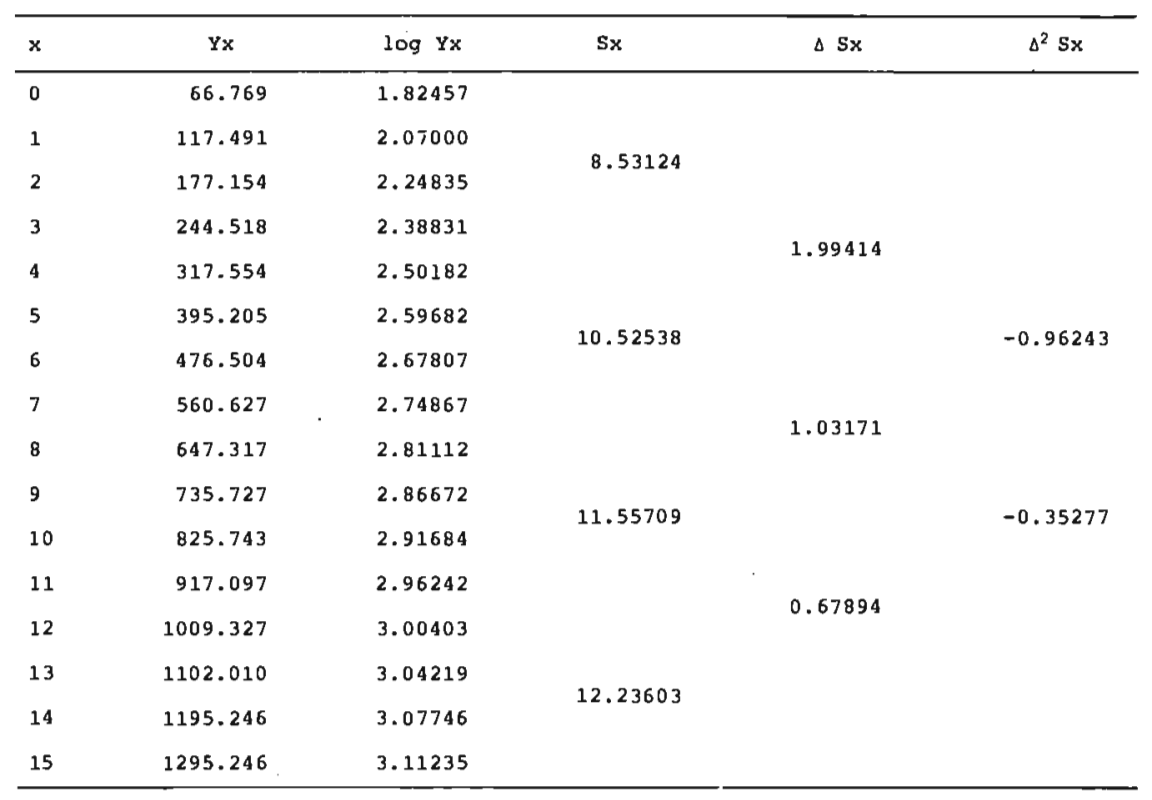

$\mathrm{d}^{\mathrm{m}}=\frac{\mathrm{S}}{\mathrm{S}}, \log \mathrm{b}=\frac{\mathrm{d}-1}{\left(\mathrm{~d}^{\mathrm{m}}-1\right)} \Delta^{2} \mathrm{~S}_{0}$ y $\mathrm{m}^{2} \log a=\Delta \mathrm{S}_{0}-\frac{\Delta^{2} \mathrm{~S}_{0}}{\mathrm{~d}^{\mathrm{m}}-1}$

$\therefore \mathrm{d}^{4}=\frac{-.35277}{-.96243}=0.36654 \longrightarrow \mathrm{d}=0.77809$

$\log b=\frac{-.77809-1}{(.36654-1)}(-.96243)=-0.84021 \longrightarrow \mathrm{b}=0.14447$

$\log a=\frac{1}{16} 1.99414-\frac{(-0.96243)}{(.36654-1)}=0.02968 \longrightarrow a=1.07072$

Teniendo la serie de valores $\hat{Y}(x)$, que en este caso representan los valores acumulados $\hat{\ell}(\mathrm{x})$, del final de la tabla al inicio de ella, se desacumulan obteniendo los valores estimados, vía la función de Makeham, de la serie $\ell(x)$ (ver Cuadro 19).

Finalmente, con la función de Makeham obtenida [ $\mathrm{Y}(\mathrm{x})=(487$. 38580) $(1.07072)^{x}(0.14447)^{(0.77809) x}$ ] se evaluó para $x=0.0$, $0.2,0.4, \ldots, 14.6,14.8,15.0$, obteniendo con ello la desagregación de la función $\ell(\mathrm{x})$. En el cuadro 20 se resumen los pasos seguidos para 
cuadro 17

\begin{tabular}{rccccccr}
\hline $\mathrm{X}$ & $\mathrm{a}^{\mathrm{X}}$ & $\mathrm{d}^{\mathrm{X}}$ & $\mathrm{b}^{\mathrm{d}}$ & $\mathrm{V}_{\mathrm{X}}$ & \multicolumn{1}{c}{$\mathrm{Y}_{\mathrm{X}}$} & \multicolumn{1}{c}{$\mathrm{YX}_{\mathrm{XX}}$} & \multicolumn{1}{c}{$\mathrm{Y}_{\mathrm{X}}$} \\
\hline 0 & 1.00000 & 1.00000 & 1.14447 & 0.14447 & 66.769 & 9.64612 & 70.41263 \\
1 & 1.07072 & 0.77809 & 0.22194 & 0.23764 & 117.491 & 27.92056 & 115.82236 \\
2 & 1.14644 & 0.60542 & 0.30996 & 0.35535 & 177.154 & 62.95167 & 173.19254 \\
3 & 1.22752 & 0.47107 & 0.40197 & 0.49343 & 244.518 & 120.65252 & 240.49078 \\
4 & 1.31433 & 0.36654 & 0.49207 & 0.64207 & 317.554 & 205.37487 & 315.21189 \\
5 & 1.40728 & 0.28520 & 0.57593 & 0.81049 & 395.205 & 320.30970 & 395.02132 \\
6 & 1.50680 & 0.22191 & 0.65095 & 0.98085 & 476.504 & 467.37895 & 478.05236 \\
7 & 1.61336 & 0.17267 & 0.71601 & 1.15518 & 560.627 & 647.62510 & 563.01833 \\
8 & 1.72746 & 0.13435 & 0.77111 & 1.33206 & 647.317 & 862.26508 & 649.22713 \\
9 & 1.84962 & 0.10454 & 0.81689 & 1.51094 & 735.727 & 1111.63935 & 736.41070 \\
10 & 1.98043 & 0.08134 & 0.85439 & 1.69206 & 825.743 & 1397.20670 & 824.68602 \\
11 & 2.12048 & 0.06329 & 0.88475 & 1.87609 & 917.097 & 1720.55651 & 914.37963 \\
12 & 2.27044 & 0.04924 & 0.90913 & 2.06413 & 1009.327 & 2083.38214 & 1006.02765 \\
13 & 2.43101 & 0.03832 & 0.92854 & 2.25729 & 1102.010 & 2487.55615 & 1100.17109 \\
14 & 2.60293 & 0.02981 & 0.94396 & 2.45706 & 1195.246 & 2936.79114 & 1197.53615 \\
15 & 2.78701 & 0.02320 & 0.95611 & 2.66469 & 1295.246 & 3451.42906 & 1298.73207 \\
& & & & & & 17912.68562 &
\end{tabular}

$\Sigma V_{x}^{2}=36.75258$

$K=\frac{\sum Y_{X} V_{X}}{\sum V x^{2}}=487.38580 \quad Y(X)=(487.38580)(1.07072)^{x}(0.14447)(0.77809)^{X}$

\begin{tabular}{|c|c|c|c|}
\hline \multicolumn{4}{|c|}{ Cuadro 18} \\
\hline $\mathrm{x}$ & $Y_{\mathbf{x}}$ & $\hat{\mathbf{Y}} \mathbf{X}$ & $\hat{\ell}_{\mathrm{x}}$ \\
\hline 0 & 66.769 & 70.41263 & \\
\hline 1 & 117.491 & 115.82236 & 45.40973 \\
\hline 2 & 177.154 & 173.19254 & 57.37018 \\
\hline & & & 67.29824 \\
\hline 3 & 244.518 & 240.49078 & 7472111 \\
\hline 4 & 317.554 & 315.21189 & 14.12111 \\
\hline 5 & 395.205 & 395.02132 & 79.80943 \\
\hline & & & 83.03104 \\
\hline 6 & 476.504 & 478.05236 & 84.96597 \\
\hline 7 & 560.627 & 563.01833 & 0620000 \\
\hline 8 & 647.317 & 649.22713 & 86.20880 \\
\hline 9 & 735.727 & 736.41070 & 87.18357 \\
\hline & & & 88.27532 \\
\hline 10 & 825.743 & 824.68602 & 89.69361 \\
\hline 11 & 917.097 & 914.37963 & 91.64802 \\
\hline 12 & 1009.327 & 1006.02765 & \\
\hline 13 & 1102.010 & 1100.17109 & 94.14344 \\
\hline 14 & 1105245 & 119753615 & 97.14344 \\
\hline 14 & 1190.240 & 1197.51615 & 101.19592 \\
\hline 15 & 1295.246 & 1298.73207 & \\
\hline
\end{tabular}


Cuadro 19

México: Distribución de la función $\ell(x)$ usando la función

de Makeham. Hombres, 1975.

\begin{tabular}{ccc}
\hline Edad & $\begin{array}{c}\ell(x) \\
\text { observadas }\end{array}$ & $\begin{array}{c}\ell(x) \\
\text { egtimadas }\end{array}$ \\
\hline 0 & 100.000 & 101.19592 \\
5 & 93.236 & 97.36506 \\
10 & 92.683 & 94.14344 \\
15 & 92.230 & 91.64802 \\
20 & 91.354 & 89.69361 \\
25 & 90.016 & 88.27532 \\
30 & 88.410 & 87.18357 \\
35 & 86.690 & 86.20880 \\
40 & 84.123 & 84.96597 \\
45 & 81.299 & 83.03104 \\
50 & 77.651 & 79.80943 \\
55 & 73.036 & 74.72111 \\
60 & 67.364 & 67.28824 \\
65 & 59.663 & 57.37018 \\
70 & 50.722 & 45.40973 \\
\hline
\end{tabular}

obtener los valores $R x$, los cuales representan los valores $\ell(x)$ con un radix de $R(15)=20.57743$. Con la serie $R x$ y tomando $\ell(x)=100000$ se obtuvo la desagregación de la función $\ell(x)$ que se muestra numériricamente, en el cuadro 21 y gráficamente en la gráfica 4.

\subsection{El sistema logito de W. Brass. ${ }^{9}$}

E1 sistema logito se basa en la siguiente relación:

$$
\operatorname{logit}(1-\ell \mathrm{x})=\alpha+\beta \operatorname{logit}\left(1-\ell_{\mathrm{X}}^{\mathbf{S}}\right)
$$

donde $\ell_{\mathrm{X}}^{\mathrm{S}}$ son los sobrevivientes a edad $\mathrm{x}$ de una tabla estándar de vida $\left(\ell_{0}=1\right.$ y $\left.\ell_{0}^{s}=1\right)$.

Se define el logito como:

$$
\operatorname{logit}(1-\ell \mathrm{x})=\frac{1}{2} \log _{e} \frac{1-\ell \mathrm{x}}{\ell \mathrm{x}}
$$

9 Ver: Brass, W.: "Sobre la escala de la mortalidad". CELADE, Serie DS No. 7, 1971. Traducción del artículo "On the scale of Mortality", incluido en Biological Aspects of Demography. Taylor and Francis Ltd., London, 1970. 
Cuadro 20

Mexico: obtencior de log valores $R_{x}$ via la funcion de Makeham.

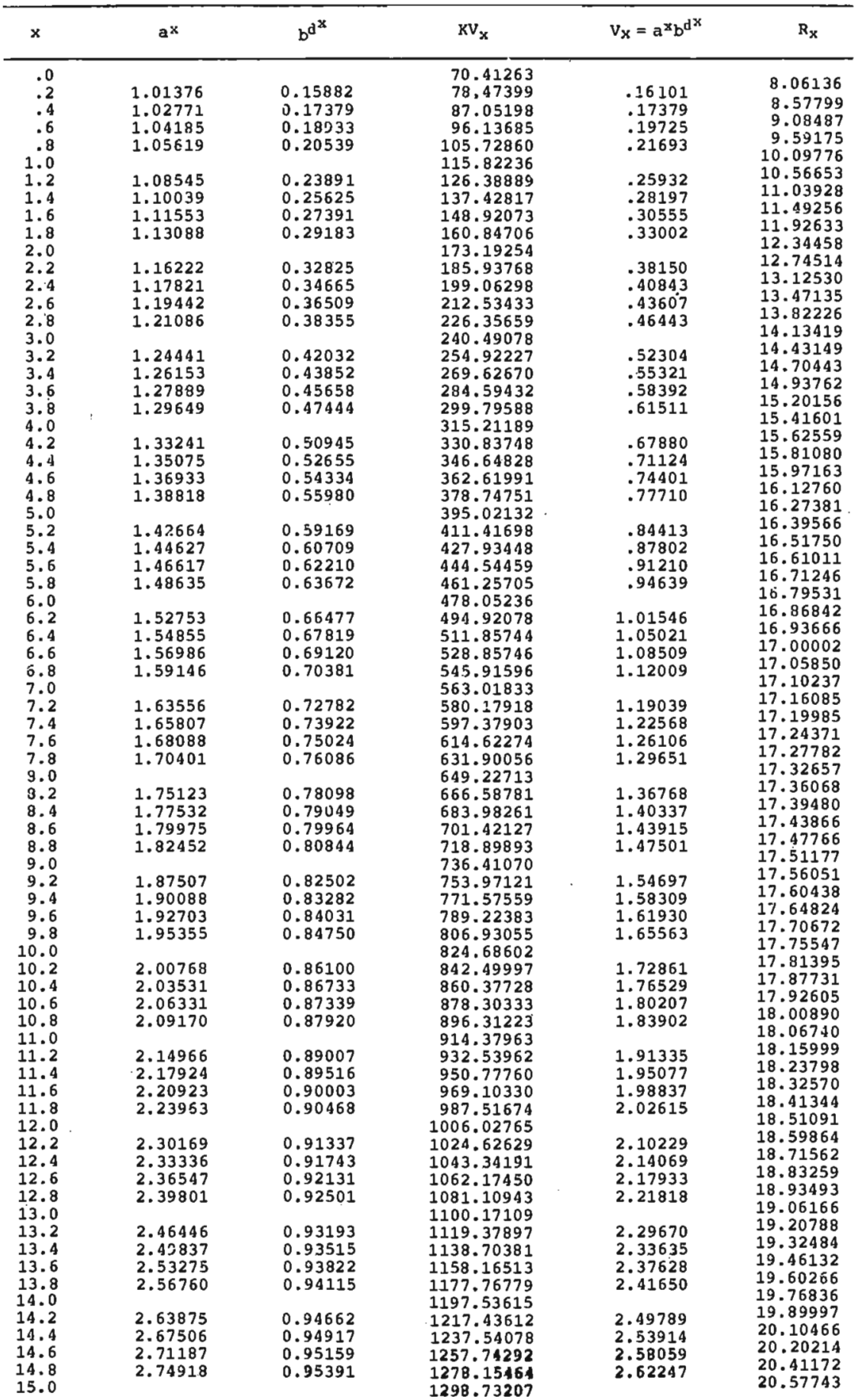


Cuadro 21

México: Desagregación de la funcion $\ell(x)$ vił la funcion de Makeham; hombres, 1975.

\begin{tabular}{|c|c|c|c|c|c|}
\hline$\underset{x}{\text { Edad }}$ & $2(x)$ & $\underset{\mathbf{x}}{\text { Edad }}$ & $\ell(x)$ & $\underset{\mathbf{x}}{\text { Edad }}$ & $\ell(x)$ \\
\hline 0 & 100.000 & 25 & 86.286 & 50 & 79.086 \\
\hline 1 & 99.195 & 26 & 85.049 & 51 & 78.375 \\
\hline 2 & 98.176 & 27 & 85.765 & 52 & 77.617 \\
\hline 3 & 97.702 & 28 & 85.552 & 53 & 76.836 \\
\hline 4 & 96.708 & 29 & 85.339 & 54 & 75.936 \\
\hline 5 & 96.068 & 30 & 85.102 & 55 & 74.917 \\
\hline 6 & 95.263 & 31 & 84.936 & 56 & 73.875 \\
\hline 7 & 94.576 & 32 & 84.747 & 57 & 72.595 \\
\hline 8 & 93.913 & 33 & 84.533 & 58 & 71.459 \\
\hline 9 & 93.344 & 34 & 84.368 & 59 & 70.133 \\
\hline 10 & 92.634 & 35 & 84.202 & 60 & 68.688 \\
\hline 11 & 92.018 & 36 & 83.965 & 61 & 67.172 \\
\hline 12 & 91.521 & 37 & 83.799 & 62 & 65.467 \\
\hline 13 & 90.952 & 38 & 83.586 & 63 & 62.785 \\
\hline 14 & 90.384 & 39 & 83.396 & 64 & 61.937 \\
\hline 15 & 89.957 & 40 & 83.112 & 65 & 59.995 \\
\hline 16 & 89.484 & 41 & 82.899 & 66 & 57.958 \\
\hline 17 & 89.057 & 42 & 82.615 & 67 & 55.850 \\
\hline 18 & 88.631 & 43 & 82.307 & 68 & 53.648 \\
\hline 19 & 88.252 & 44 & 81.975 & 69 & 51.350 \\
\hline 20 & 87.802 & 45 & 81.620 & 70 & 49.072 \\
\hline 21 & 87.518 & 46 & 81.217 & 71 & 46.613 \\
\hline 22 & 87.115 & 47 & 80.720 & 72 & 44.150 \\
\hline 23 & 86.878 & 48 & 80.270 & 73 & 41.686 \\
\hline 24 & 86.570 & 49 & 79.678 & 74 & 39.176 \\
\hline
\end{tabular}

La relación (137) señala que existe una relación lineal entre la serie de los logitos observados [logit $(1-\ell \mathrm{x})$ ] y la serie de los logitos del estándar [logit $(1-\ell \mathbf{X})]$.

La relación (138) surge a partir de la siguiente función:

$$
f(x)=-\frac{1}{2} \operatorname{sech}^{2} \frac{x-\alpha}{\beta}
$$

que corresponde a una curva simétrica cuya medida es $\alpha$ y cuyo desvío estándar es igual a $\beta$. Esta curva es una curva de respuesta usada en ensayos biológicos y sirve para estimar cómo los animales reaccionan a una cierta droga: por analogía se piensa en una población humana y la forma en que muere a medida que aumenta la dosis de tiempo.

Sea $Y_{S}(x)$ el logit $\left(1-\ell x^{S}\right)$ y $Y(x)$ el logito obtenido directamente con los valores observados: logit $(1-\ell x)$. Se tiene una tabla están- 


\section{Gráfica 4}

México: Distribución de la función $l(x)$, desagregada a través de la función de Makeham, hombres, 1975.

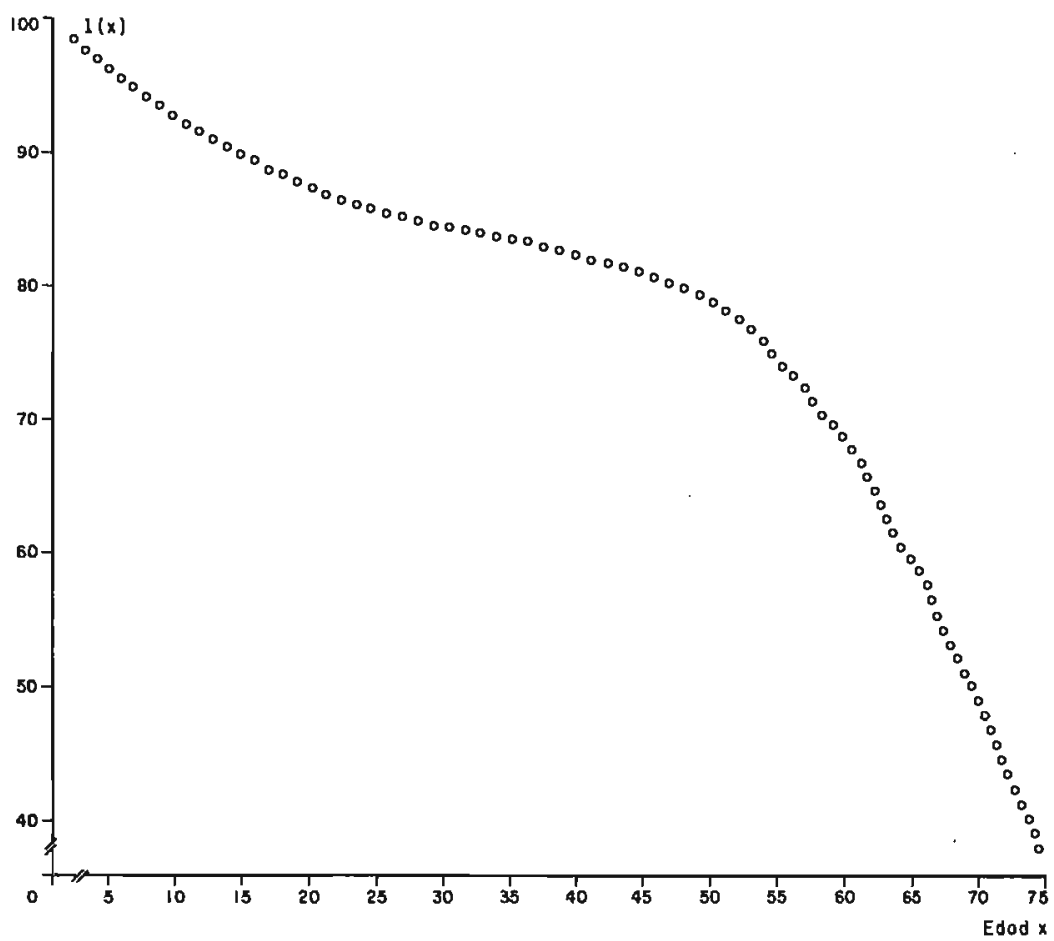

dar de mortalidad, con la cual se calculan los valores de la serie $Y_{S}(x)$ y una tabla empírica con la cual se calculan los valores de la serie $\mathrm{Y}(\mathrm{x})$.

Después se grafican los valores $\mathrm{Y}_{\mathrm{S}}(\mathrm{x})$ contra los $\mathrm{Y}(\mathrm{x})$ observándose la relación lineal que guardan. En el caso de tablas con valores de $\ell(x)$ para $\mathrm{x}=0,1,5,10,15, \ldots, 70,75$ y 80 , que generan 18 puntos de la forma $\left[Y_{S}(x), Y(x)\right]$, se llevan a cabo los siguientes pasos para obtener la recta de ajuste:

1) Se dividen los 18 puntos en dos grupos, de 9 observaciones cada uno, el primero para $x=0,1,5, \ldots, 30$ y 35 y el segundo para $x=40$, $45, \ldots, 75$ y 80 .

Nota: De hecho el primer grupo consta sólo de 8 puntos ya que $\ell(0)$ $=1 \mathrm{y}$ el logito para $\mathrm{x}=0$ no está definido. 
2) Se calculan los puntos medios:

$\mathrm{P}_{1}=\overline{\mathrm{Y}}_{1}^{S}(\mathrm{x}), \overline{\mathrm{Y}}_{1}(\mathrm{x}) \quad \mathrm{y} \quad \mathrm{P}_{2}=\overline{\mathrm{Y}}_{2}^{\mathrm{S}}(\mathrm{x}), \overline{\mathrm{Y}}_{2}(\mathrm{x})$

donde:

$\bar{Y}(x)=\frac{\sum_{x=1}^{35} Y(x)}{8}$

$\bar{Y}_{1}^{S}(x)=\frac{\sum_{x=1}^{35} Y^{S}(x)}{8}$

y

$\bar{Y}_{2}(x)=\frac{\sum_{x=40}^{80} Y(x)}{9} \quad Y_{2}^{S}(x)=\frac{\sum_{x=40}^{80} Y S(x)}{9}$

3) Se obtiene la recta de ajuste que pasa por los puntos $P_{1}$ y $P_{2}$ :

$$
\mathrm{Y}(\mathrm{x})-\overline{\mathrm{Y}}_{1}(\mathrm{x})=\frac{\overline{\mathrm{Y}}_{2}(\mathrm{x})-\overline{\mathrm{Y}}_{1}(\mathrm{x})}{\overline{\mathrm{Y}}_{1}^{\mathrm{S}}(\mathrm{x})-\overline{\mathrm{Y}}_{1}^{\mathrm{S}}(\mathrm{x})} \mathrm{Y}_{\mathrm{S}}(\mathrm{x})-\overline{\mathrm{Y}}_{1}^{\mathrm{S}}(\mathrm{x})
$$

donde:

$$
\beta=\frac{\bar{Y}_{2}(x)-\bar{Y}_{1}(x)}{\bar{Y}_{2}^{S}(x)-\bar{Y}_{1}^{S}(x)}
$$

Sustituyendo (144) en (143)

$$
\begin{aligned}
\mathrm{Y}(\mathrm{x})-\overline{\mathrm{Y}}_{1}(\mathrm{x}) & =\beta \mathrm{Y}_{\mathrm{S}}(\mathrm{x})-\mathrm{Y}_{1}^{\mathrm{S}}(\mathrm{x}) \\
\mathrm{Y}(\mathrm{x}) & =\beta \mathrm{Y}_{\mathrm{s}}(\mathrm{x})+\overline{\mathrm{Y}}_{1}(\mathrm{x})-\beta \overline{\mathrm{Y}}_{1}^{\mathrm{S}}(\mathrm{x})
\end{aligned}
$$

donde:

$$
\alpha=\bar{Y}_{1}(x)-\beta \bar{Y}_{1}^{S}(x)
$$

Por lo tanto:

$$
\mathrm{Y}(\mathrm{x})=\alpha+\beta \mathrm{Y}_{\mathrm{S}}(\mathrm{x})
$$


4) Obtenida la recta de ajuste (148), se calculan los valores de los logitos ajustados $[\widehat{Y}(\mathrm{x})]$, sustituyendo en la relación (148) los valores de los logitos estándar.

5) Se estima la serie ajustada de valores $\hat{\ell}(x)$, aplicando los antilogitos (153).

Sabemos:

$$
\mathrm{Y}(\mathrm{x})=\frac{1}{2} \log _{e} \frac{1-\hat{\ell}(\mathrm{x})}{\hat{\ell}(\mathrm{x})}
$$

entonces:

$$
\begin{aligned}
& 2 \hat{Y}(x)=\log _{e} \frac{1-\hat{\ell}(x)}{\hat{\ell}(x)} \\
& \exp 2 \hat{Y}(x)=\frac{1-\hat{\ell}(x)}{\hat{\ell}(x)} \\
& \hat{\ell}(x) \cdot \exp [2 \hat{Y}(x)]+\hat{\ell}(x)=1 \\
& \hat{\ell}(x)=\frac{1}{1+\exp [2 \hat{Y}(x)]}
\end{aligned}
$$

6) Finalmente se calculan los cocientes de mortalidad:

$$
q(x)=\frac{\ell(x)-\ell(x+1)}{\ell(x)}
$$

Ejemplo de la aplicación del sistema logito: obtención de la tabla de mortalidad corregida para la República Mexicana, hombres, 1975.

Se obtuvieron los valores de los logitos observados y los del están$\operatorname{dar}[\mathrm{Y}(\mathrm{x})$ y $\mathrm{Y}(\mathrm{x})]$, ver cuadro 23.

Se calculan los puntos medios $P_{1}$ y $P_{2}$ (empleando las relaciones (1.40) y (141):

$P_{1}=(-1.17545,-1.18790)$ y $P_{2}=(-0.30503,-0.28419)$

siendo la recta que pasa por $P_{1}$ y $P_{2}$ (empleando las relaciones (144) y (147): 
Cuadro 22

México: Valores $\ell_{x}$ y $\ell_{x}^{\mathfrak{s}}$, nivel nacional, hombres, 1975.

\begin{tabular}{ccc}
\hline Edad & $\ell_{\mathbf{x}}$ a/ & $\ell_{\mathbf{x}}^{\mathrm{s}} \underline{\mathrm{b} /}$ \\
\hline 0 & 1.00000 & 1.00000 \\
1 & 0.94822 & 0.94496 \\
5 & 0.93236 & 0.92939 \\
10 & 0.92683 & 0.92305 \\
15 & 0.92230 & 0.91818 \\
20 & 0.91354 & 0.90997 \\
25 & 0.90016 & 0.89849 \\
30 & 0.88410 & 0.88671 \\
35 & 0.86690 & 0.87357 \\
40 & 0.83123 & 0.85742 \\
45 & 0.81299 & 0.83576 \\
50 & 0.77651 & 0.80507 \\
55 & 0.73036 & 0.76109 \\
60 & 0.57364 & 0.69856 \\
65 & 0.59663 & 0.61258 \\
70 & 0.50722 & 0.50076 \\
75 & 0.39615 & 0.36521 \\
80 & 0.27154 & 0.22162 \\
\hline
\end{tabular}

Puentes: a/ SSA, Subsecretarla de planeación, dirección general de bioestadistica, serie bioestadistica, No. 2 "Tablas abreviadas de mortalidad por estados y para ocho regiones de México", 1980.

b/ Valores obtenidos de las tablas modelo de Coale y Demeny, región Oeste; a dichas tablas se entrố con la esperanza de vida al nacimiento observada para los hombres en 1975 (nivel nacional). Interpolando dicho valor se obtuvo la tabla estándar.

Cuadro 23

MExico: Valores de los logitos $Y(x)$ y $Y_{S}(x)$, nivel nacional, hombres, 1975.

\begin{tabular}{rrr}
\hline Edad & $Y(x)$ & $Y_{S}(x)$ \\
\hline 1 & -1.45379 & -1.42154 \\
5 & -1.31176 & -1.28868 \\
10 & -1.26949 & -1.21226 \\
15 & -1.23701 & -1.20894 \\
20 & -1.17882 & -1.15663 \\
25 & -1.09950 & -1.09028 \\
30 & -1.01592 & -1.02878 \\
35 & -0.93691 & -0.96645 \\
40 & -0.83370 & -0.89701 \\
45 & -0.73478 & -0.81351 \\
50 & -0.62272 & -0.70914 \\
55 & -0.49822 & -0.57933 \\
60 & -0.36235 & -0.42023 \\
65 & -0.19572 & -0.22909 \\
70 & -0.01444 & -0.00152 \\
75 & 0.21077 & 0.27641 \\
80 & 0.49341 & 0.62813 \\
\hline
\end{tabular}




$$
\hat{\mathrm{Y}}(\mathrm{x})=(0.03251)+(1.03825) \mathrm{Y}^{\mathrm{s}}(\mathrm{x})
$$

En la gráfica 5 se observa la distribución lineal de los puntos... $\left[Y^{S}(x), Y(x)\right]$ así como la recta de ajuste para el caso estudiado.

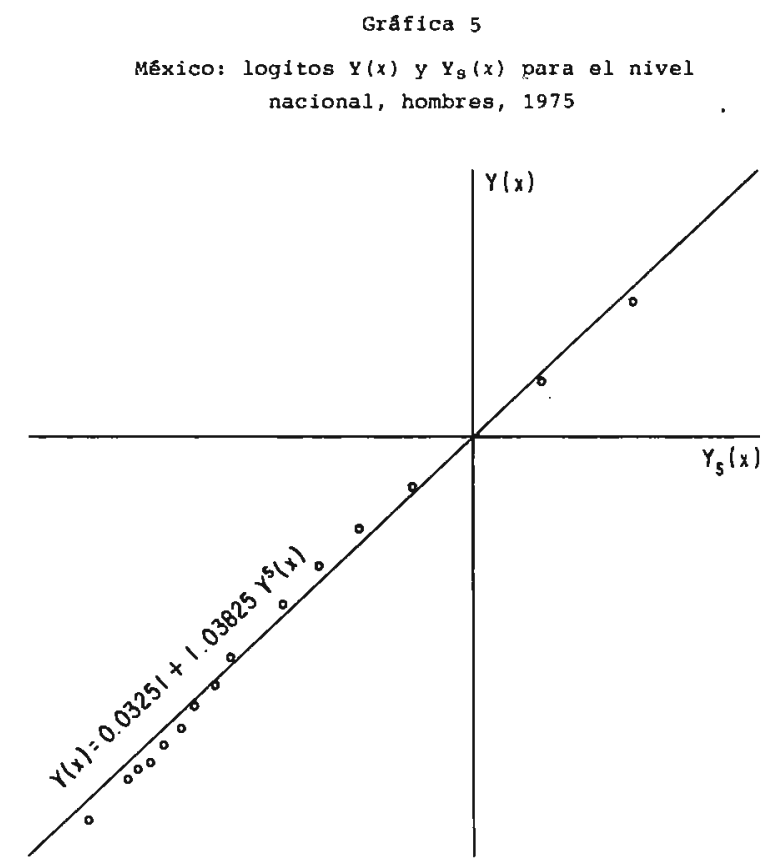

Obtenida la recta $\mathrm{Y}(\mathrm{x})=0.3251+1.03825 \mathrm{YS}^{\mathrm{s}}(\mathrm{x}) \ldots$ (c) se calculan los valores de los logitos ajustados $[\hat{\mathrm{Y}}(\mathrm{x})]$, sustituyendo en la relación (c) los valores de los logitos estándar; después se obtienen los valores êx aplicando los antilogitos (ver Cuadro 24).

Obtenidos los valores $\ell$ x se pasó a calcular la tabla de mortalidad corregida para la República Mexicana, hombres, 1975. En el cuadro 25 se dan los valores de las series $\mathrm{q}(\mathrm{x})$, observadas y estimadas.

\section{Presentación de los ajustes obtenidos}

\subsection{Nupcialidad}

Se aplicó el modelo estándar de nupcialidad de A. J. Coale y e] modelo bilogístico de $\mathrm{A}$. Bocaz a datos de las encuestas PECFAL- 
Cuadro 24

México: Valores $\hat{\ell}_{x}$ para el nivel nacional, hombres, 1973.

\begin{tabular}{ccc}
\hline Edad & $\hat{\mathrm{Y}}(\mathrm{x})$ & $\hat{\ell} \mathrm{x}$ \\
\hline 0 & -1.44340 & 1.00000 \\
1 & -1.30546 & 0.94718 \\
5 & -1.25727 & 0.93156 \\
10 & -1.22267 & 0.92515 \\
15 & -1.16836 & 0.92022 \\
20 & -1.09947 & 0.91187 \\
25 & -1.03562 & 0.90015 \\
30 & -0.97091 & 0.88808 \\
35 & -0.89881 & 0.87455 \\
40 & -0.81212 & 0.85786 \\
45 & -0.70375 & 0.83538 \\
50 & -0.56898 & 0.80337 \\
55 & -0.40379 & 0.75730 \\
60 & -0.20534 & 0.69159 \\
65 & 0.03093 & 0.60123 \\
70 & 0.31949 & 0.48454 \\
75 & 0.68467 & 0.34548 \\
80 & & 0.20273 \\
\hline & &
\end{tabular}

RURAL (Encuesta Comparativa de Fecundidad en América Latina, zonas rurales 1970) y EMF (Encuesta Mexicana de Fecundidad, 1976); tomando a la población femenina por edad a la primera unión para las cohortes 15-19 años, 20-24 años, ..., 40-44 años y 45-49 años.

Con el fin de observar los tipos de ajuste que se obtienen al aplicar las dos técnicas antes mencionadas se tomó la cohorte 35-39 años de edad, tanto de la PECFAL-RURAL como de la EMF; los resultados de la aplicación se observan en los cuadros 26 y 27 y los ajustes obtenidos se muestran en las gráficas 6 y 7 .

Se observa que el modelo de Albino Bocaz no considera ningún estándar como referencia para ajustar la estructura por edad de las primeras uniones, el modelo de Coale sí lo considera siendo el estándar de Coale universal; a simple vista (ver gráficas 6 y 7 ) el modelo de Bocaz nos da un mejor ajuste que el de Coale, esto bajo el supuesto de que la información observada estuviese bien detectada, lo cual no es cierto; por esto último el modelo que realmente corrige la información estrictamente hablando, es el modelo de A.J. Coale. 
Cuadro 25

MExico: Valores $g(x)$, observados y ajustados, para el nivel nacional, nombres, 1975.

\begin{tabular}{ccc}
\hline Edad & $\mathrm{q}(\mathrm{x})$ & $\hat{\mathrm{q}}(\mathrm{x})$ \\
\hline 0 & 0.05178 & 0.05281 \\
1 & 0.01672 & 0.01649 \\
5 & 0.00593 & 0.00688 \\
10 & 0.00489 & 0.00533 \\
15 & 0.00950 & 0.00908 \\
20 & 0.01464 & $0.0128 j$ \\
25 & 0.01784 & 0.01342 \\
30 & 0.01946 & 0.01523 \\
35 & 0.02961 & 0.01909 \\
40 & 0.03358 & 0.02621 \\
45 & 0.04487 & 0.03831 \\
50 & 0.05943 & 0.05735 \\
55 & 0.07766 & 0.08677 \\
60 & 0.11432 & 0.13064 \\
65 & 0.14986 & 0.19411 \\
70 & 0.21898 & 0.28701 \\
75 & 0.31455 & 0.41319 \\
80 & 1.00000 & 1.00000 \\
\hline
\end{tabular}

En resumen, el modelo de Albino Bocaz está basado en la información observada y vía una transformación lineal suaviza dichas observaciones, en cambio el modelo de Coale si bien es cierto que también se basa en las observaciones dadas, involucra un estándar de nupcialidad universal el cual realmente corrige la distribución por edad de la nupcialidad.

Viendo por separado cada uno de los ajustes, es decir, por una parte los ajustes obtenidos para la cohorte 35-39 vía el modelo de Bocaz, tanto para la PECFAL-RURAL como para la EMF, y por otro lado los ajustes obtenidos al aplicar el modelo de Coale a esa misma cohorte y para las mismas encuestas, observamos coherencia en los resultados, lo cual se puede ver en las gráficas 8 (la cual muestra los ajustes vía el modelo de Bocaz) y 9 (la cual muestra los ajustes de Coale). Dichas gráficas nos explican el peligro que puede haber at pensar que el modelo de Bocaz corrige, estrictamente hablando, la estructura por edad de los primeros matrimonios que como ya se vio es falso. 
Grâfica h
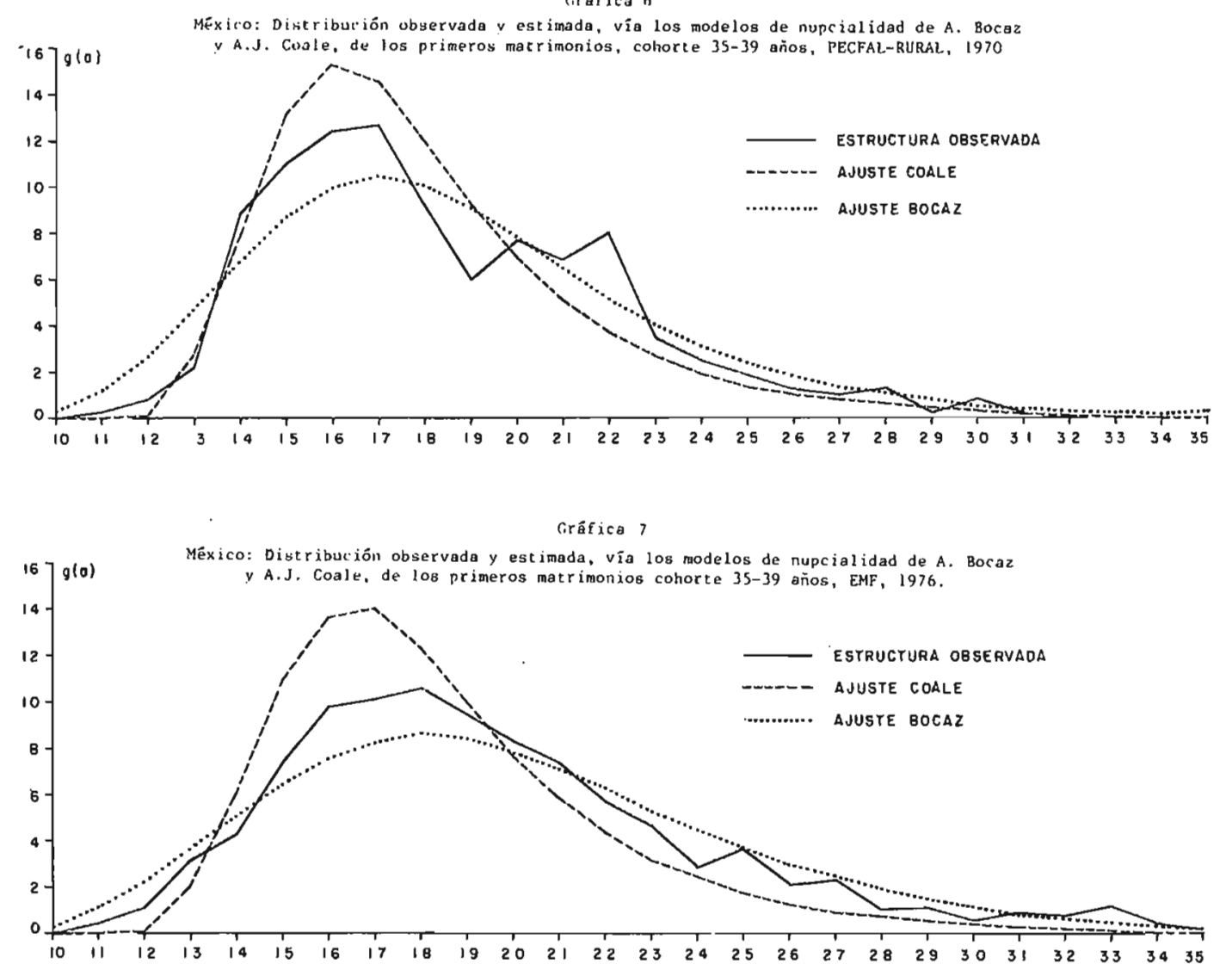


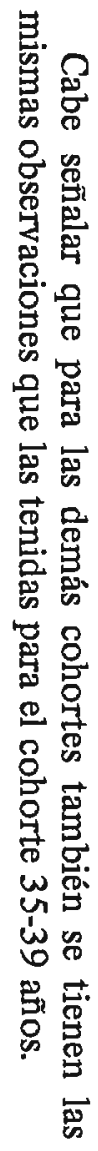

Gräfica 8

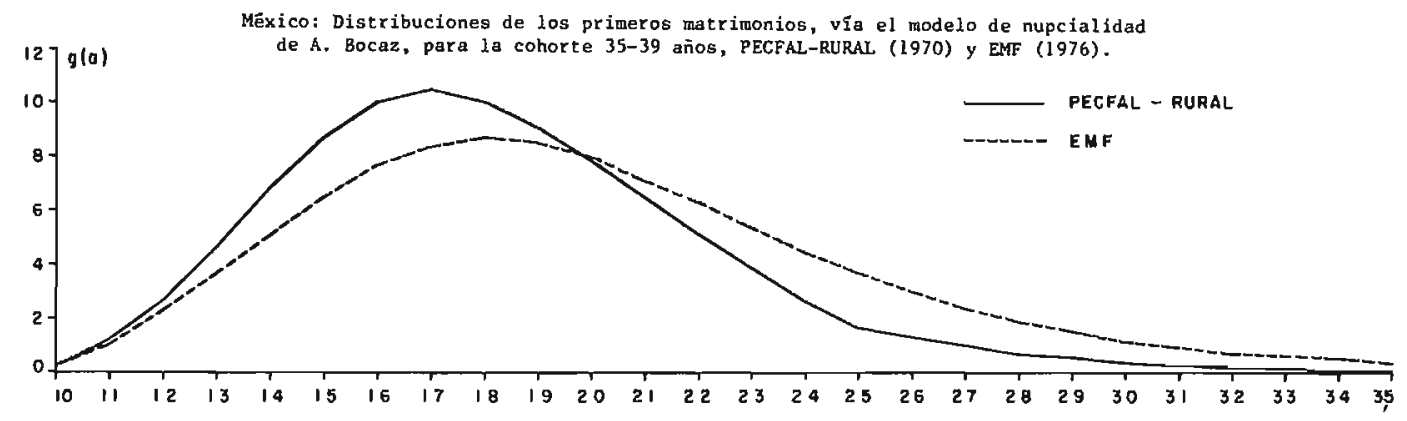

Grâfica 9 
Cuadro 26

\begin{tabular}{|c|c|c|c|}
\hline$a$ & $g^{0}(a)$ & $g(a)$ Bocaz & g(a) Coale \\
\hline 10 & 0.00 & 0.25 & 0 \\
\hline 11 & 0.28 & 1.23 & 0 \\
\hline 12 & 0.83 & 2.77 & 0.18 \\
\hline 13 & 2.21 & 4.73 & 2.69 \\
\hline 14 & 8.84 & 6.84 & 7.86 \\
\hline 15 & 11.05 & 8.72 & 13.15 \\
\hline 16 & 12.43 & 9.98 & 15.33 \\
\hline 17 & 12.71 & 10.42 & 14.58 \\
\hline 18 & 9.39 & 10.06 & 12.01 \\
\hline 19 & 6.08 & 9.09 & 9.23 \\
\hline 20 & 7.73 & 7.81 & 6.92 \\
\hline 21 & 6.91 & 6.45 & 5.08 \\
\hline 22 & 8.01 & 5.17 & 3.72 \\
\hline 23 & 3.59 & 4.07 & 2.68 \\
\hline 24 & 2.49 & 3.15 & 1.87 \\
\hline 25 & 1.93 & 2.41 & 1.31 \\
\hline 26 & 1.38 & 1.83 & 1.00 \\
\hline 27 & 1.10 & 1.38 & 0.79 \\
\hline 28 & 1.38 & 1.04 & 0.58 \\
\hline 29 & 0.27 & 0.77 & 0.41 \\
\hline 30 & 0.83 & 0.57 & 0.26 \\
\hline 31 & 0.27 & 0.41 & 0.20 \\
\hline 32 & 0.00 & 0.30 & 0.12 \\
\hline 33 & 0.00 & 0.21 & 0.00 \\
\hline 34 & 0.00 & 0.14 & 0.00 \\
\hline 35 & 0.28 & 0.09 & 0.00 \\
\hline 36 & 0.00 & 0.06 & 0.00 \\
\hline 37 & 0.00 & 0.03 & 0.00 \\
\hline 38 & 0.00 & 0.01 & 0.00 \\
\hline 39 & 0.00 & 0.00 & 0.00 \\
\hline
\end{tabular}

\subsection{Fecundidad}

Con información del censo nacional de población de 1970 y las estadísticas vitales, se calcularon las tasas específicas de fecundidad para 8 regiones de la República Mexicana (ver cuadro 28).

A cada una de las estructuras de fecundidad se les aplicó el modelo de nupcialidad de Coale y Trussell y se obtuvieron las funciones de fecundidad de Brass. Tomando los ajustes obtenidos para la región 3 , se tiene que la desagregación de las tasas específicas de fecundidad, de grupos quinquenales a edades individuales, se logro a través de los dos métodos (ver cuadro 29).

En la gráfica 10 se muestran las distribuciones de la estructura por edad de la fecundidad vía la función de fecundidad de Brass y el 
Cuadro 27

México: Estimación de los primeros matrimonios $(\mathrm{g}(a))$, a través de los modelos de nupcialidad de A. Bocaz y A. J. Coale, cohorte 35-39 años, EMF, 1976

\begin{tabular}{lrcc}
\hline$g$ & $g^{0}(a)$ & $g(a)$ Bocaz & $g(a)$ Coale \\
\hline 10 & 0.00 & 0.30 & 0.00 \\
11 & 0.46 & 1.19 & 0.00 \\
12 & 1.14 & 2.36 & 0.01 \\
13 & 3.19 & 3.72 & 2.04 \\
14 & 4.33 & 5.17 & 6.13 \\
15 & 7.41 & 6.53 & 10.93 \\
16 & 9.80 & 7.63 & 13.75 \\
17 & 10.14 & 8.36 & 14.01 \\
18 & 10.60 & 8.63 & 12.41 \\
19 & 9.46 & 8.45 & 9.99 \\
20 & 8.32 & 7.93 & 7.77 \\
21 & 7.41 & 7.15 & 5.94 \\
22 & 5.70 & 6.26 & 4.44 \\
23 & 4.68 & 5.34 & 31.34 \\
24 & 2.85 & 4.46 & 2.47 \\
25 & 3.65 & 3.67 & 1.78 \\
26 & 2.05 & 2.97 & 1.27 \\
27 & 2.28 & 2.38 & $0 ! 97$ \\
28 & 1.03 & 1.89 & 0.79 \\
29 & 1.14 & 1.48 & 0.162 \\
30 & 0.57 & 1.15 & 0.44 \\
31 & 0.91 & 0.88 & 0.29 \\
32 & 0.57 & 0.67 & 0.22 \\
33 & 1.14 & 0.49 & 0.15 \\
34 & 0.46 & 0.36 & 0.06 \\
35 & 0.23 & 0.25 & 0.00 \\
36 & 0.34 & 0.16 & 0.00 \\
37 & 0.11 & 0.10 & 0.00 \\
38 & 0.00 & 0.05 & 0.00 \\
39 & 0.00 & 0.01 & 0.00 \\
\hline
\end{tabular}

modelo de fecundidad de Coale y. Trussell; si bien es cierto que las dos distribuciones son muy semejantes existen diferencias en la obtención de cada una de ellas, las cuales son: la función de fecundidad de Brass considera sólo la estructura de fecundidad observada, con lo que la desagregación está basada en un ajuste que suaviza el comportamiento de las tasas específicas de fecundidad observadas, sin que necesariamente sea corregida dicha estructura, en cambio con el modelo de fecundidad de Coale y Trussell sí se está corrigiendo dicha estructura, que si bien se basa en la estructura observada, para estimar los parámetros $a_{\circ}, \mathrm{K} \mathrm{y} \mathrm{m}$ (ver presentación del modelo), con ellos se entra a las tablas estándar de fecundidad, las cuales desagregan y corrigen las tasas quinquenales de fecundidad. 
Cuadro 28

Mexico: Tasas especificas de fecundidad observadas para ocho regiones"/ 1970

\begin{tabular}{|c|c|c|c|c|c|c|c|c|}
\hline \multirow{2}{*}{$\begin{array}{l}\text { Grupos } \\
\text { de edad }\end{array}$} & \multirow[b]{2}{*}{ Regiốn 1} & \multirow[b]{2}{*}{ Regiôn 2} & \multicolumn{4}{|c|}{ Tasas especificad de Fecundidad } & \multirow[b]{2}{*}{ Región 7} & \multirow[b]{2}{*}{ Regiốn } \\
\hline & & & Region 3 & Regiốn 4 & Regiôn 5 & Regiôn 6 & & \\
\hline $10-14$ & .006852 & .005635 & .005980 & .006931 & .005173 & .006669 & .003363 & .007427 \\
\hline $15-19$ & .099830 & .101023 & .075881 & .108421 & .086394 & .111917 & .062422 & .116659 \\
\hline $20-24$ & .309937 & .292224 & .225701 & .339261 & .298868 & .327244 & .236672 & .305102 \\
\hline $25-29$ &. .61224 & .339958 & .252655 & .396228 & .355658 & .346320 & .277851 & .306662 \\
\hline $30-34$ & .28844 & .279880 & .225343 & .332968 & .313209 & .293992 & .232856 & .250673 \\
\hline $35-39$ & .207526 & $x x>7$ & .162822 & .257775 & .243309 & .220081 & .166772 & .186627 \\
\hline $40-49$ & .080235 & .079988 & .087711 & .090303 & .084618 & .084618 & .066380 & .073523 \\
\hline
\end{tabular}

* Regín 1: Baja Califori.ia Norte, Baja California Sur, Nayarit, Sinaloa y Sonora. Region 2: Coahuila, Chihuahua, Durango y Nuevo León.

Region 3: Tamaulipas y Veracruz.

Región 4: Aguascalientes, San Luis Potosi y Zacatecas.

Región 5: Guanajuato, Jalisco y Michoacán.

Region 6: Hidalgo, Morelos, Puebla, Querétaro y Tlaxcala.

Región 7: Distrito Federal y México.

Region 8: Guerrero, Oaxaca, Chiapas, Tabasco, Xucatán y Quintana Roo.

Cuadro 29

Estimación de la estructura por edad de la fecundidad $(f(x))$, a traves de la función de fecundidad de Brass y el modelo de fecundidad de coale y Trussell,
región 3 (Tamalipas y veracruz), 1970

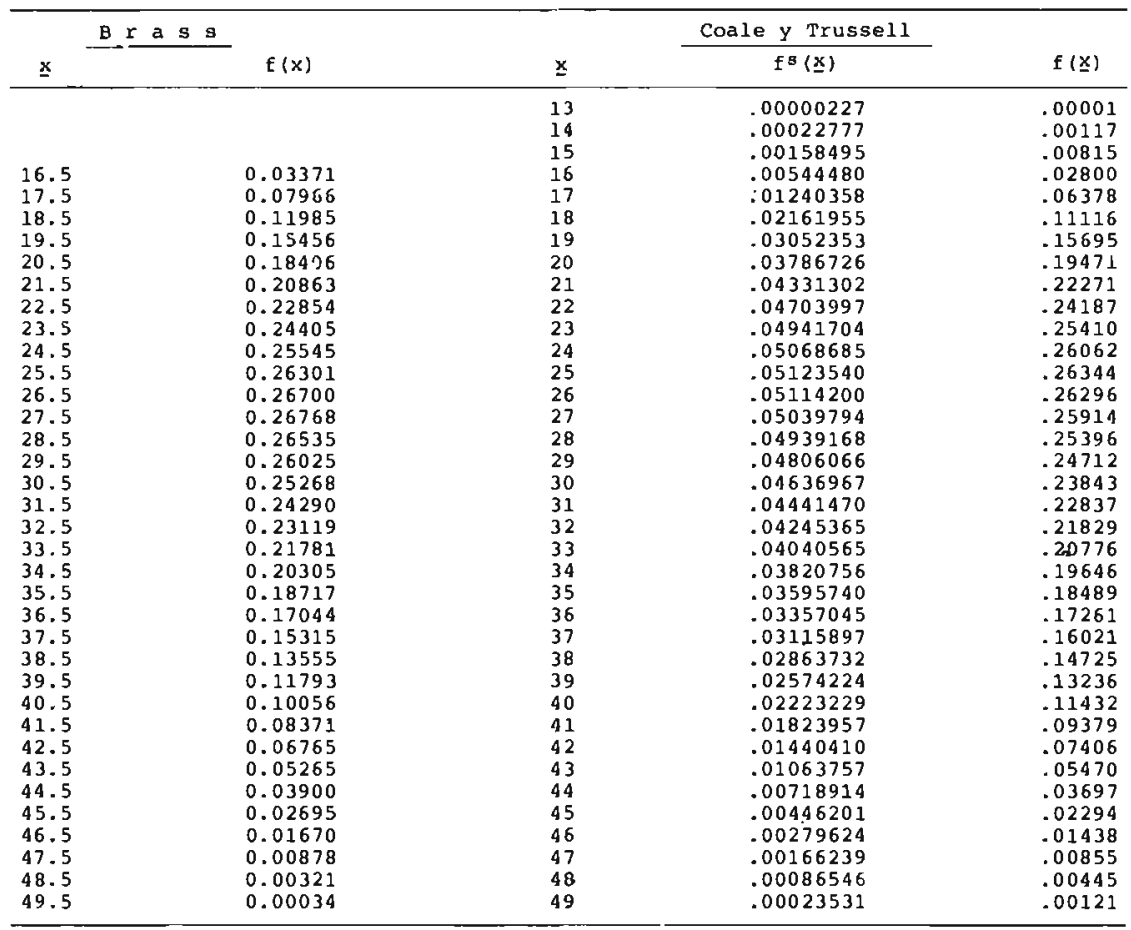




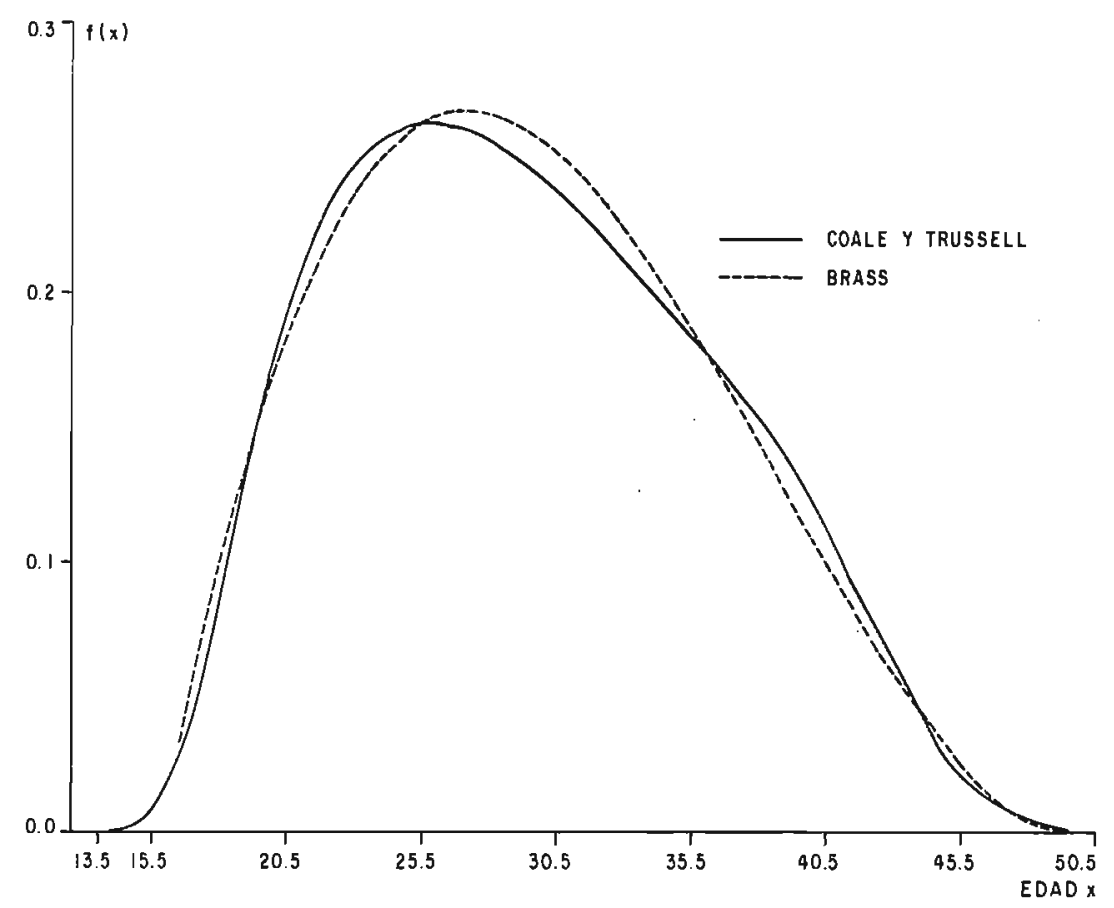

En resumen, 1a función de fecundidad de Brass desagrega y suaviza la estructura de fecundidad observada, y el modelo de Coale y Trussell desagrega y corrige dicha estructura. Cabe señalar que para estos dos modelos los ajustes obtenidos, a partir de cada uno de ellos, no difieren tanto como los obtenidos en el caso de los modelos de nupcialidad de Coale y Bocaz.

\subsection{Mortalidad}

Tomando de la tabla abreviada de mortalidad, para los hombres, à nivel nacional $1975,{ }^{10}$ las series $q(x)$ y $\ell(x)$ se aplicaron el sistema lo-

${ }^{10}$ Secretaría de Salubridad y Asistencia, op. cit., p. 19. 
gito de W. Brass (tomando como estándar la tabla modelo de Coale y Demeny, asociada a la esperanza de vida observada) y la función de Makeham, obteniendo los resultados que aparecen en el cuadro 30.

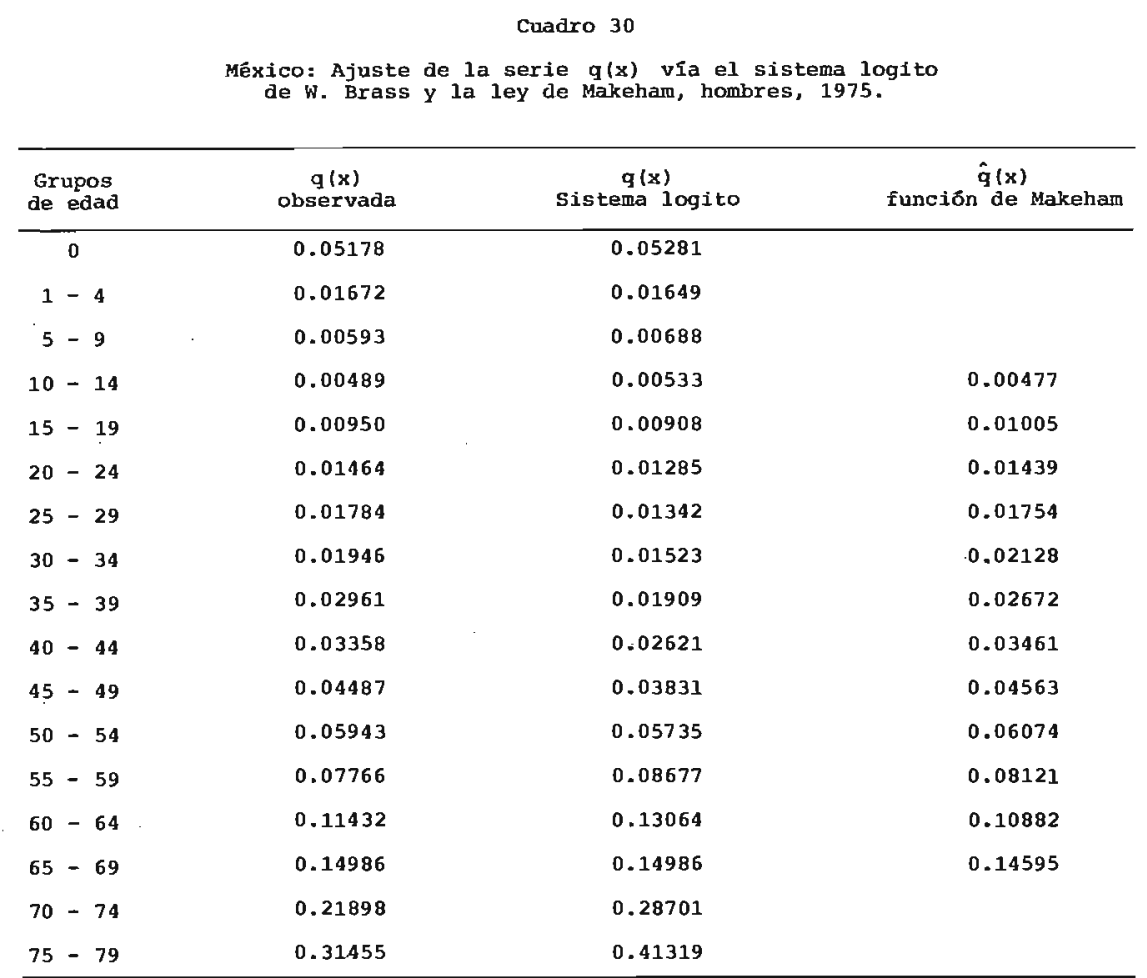

Fuente: Cuadros 15 y 25.

En las gráficas 11 y 12 se muestran la bondad de los ajustes obtenidos. Por una parte (gráfíca 11), con el sistema logito, relacionamos la experiencia de mortalidad observada con la de un estándar escogido, dicha relación se linealiza vía los logitos observados y estimados con lo cual se pasa a corregir la estructura por edad de la mortalidad. Por otra parte (gráfica 12), con la función de Makeham, caracterizamos la experiencia de la mortalidad observada, obteniendo en base a ella la forma de la función de distribución de la mortalidad.

Así, mientras que la función de Makeham nos da la distribución de la mortalidad, sin corregir los niveles que en cada grupo de edad se observa, el sistema logito de Brass corrige la estructura de la mortalidad. 
Se debe tener cuidado cuando se tenga un ajuste, como el que da la función de Makeham, tal que difiera mínimamente de los valores observados, y se piense que se está corrigiendo la estructura por edad de la mortalidad, o bien, tener un ajuste, como cl que da el sistema logito de W. Brass, que si bien puede no estar necesariamente correlacionado con los niveles observados de mortalidad, y estar corrigiendo adecuadamente la estructura por edad de la mortalidad.

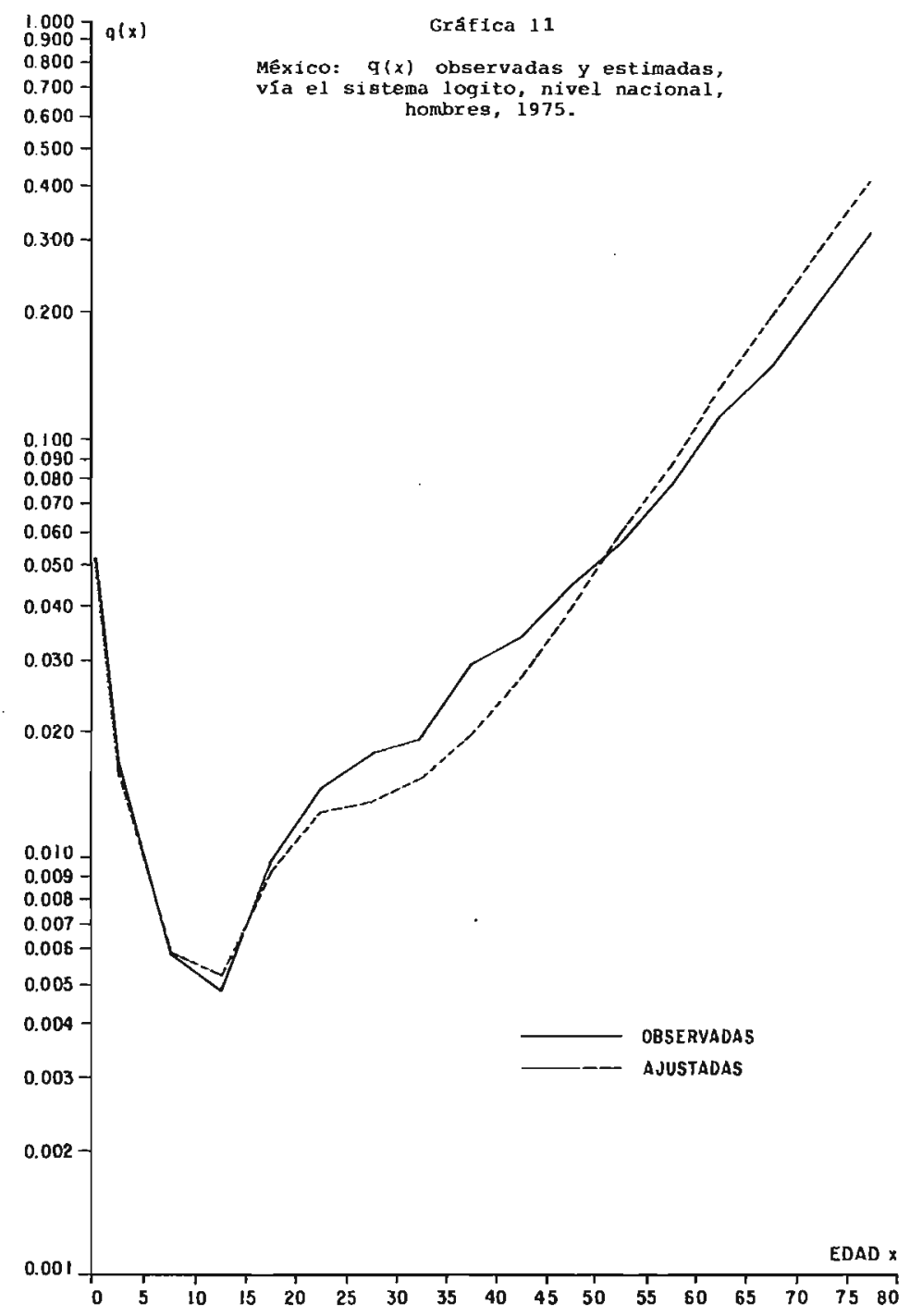


Grafica 12

México: $q(x)$ observadas y estimadas via la funcion. de Makeham, nivel nacional, hombres, 1975.

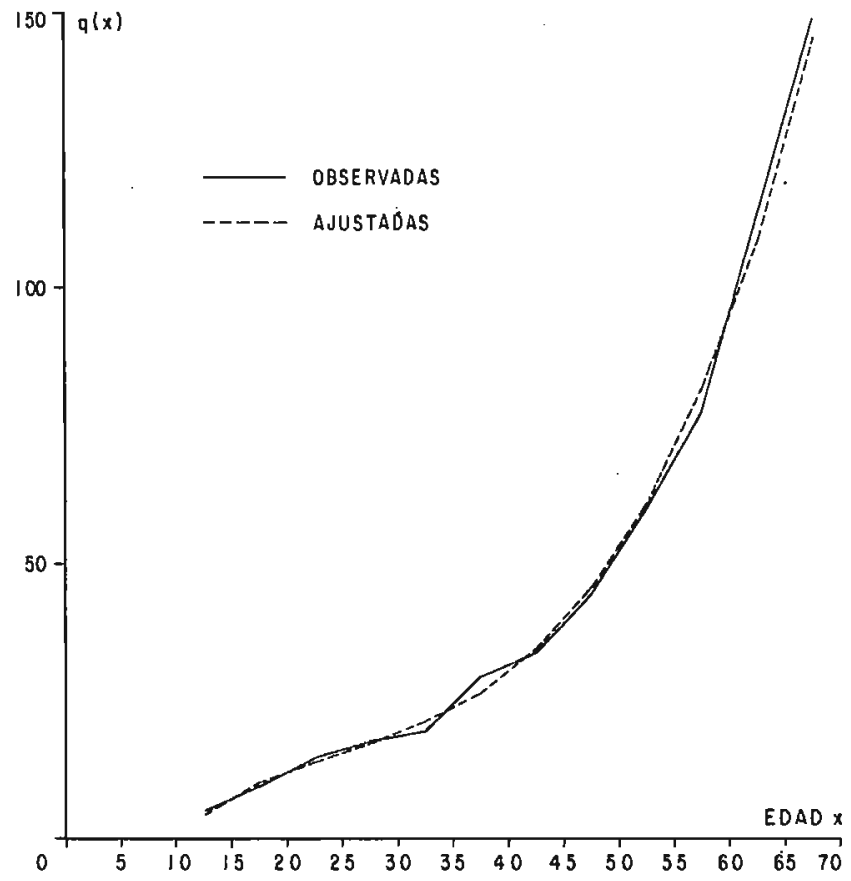

Cabe señalar que una de las ventajas que tiene la función de Makreham sobre el sistema logito es que la primera sirve para desagregar de grupos de edades quinquenales a edades individuales la experiencia de mortalidad de una población (ver ejemplo en la presentación de la función de Makeham). Para que el sistema logito sirviera para corregir y desagregar la estructura por edad de la mortalidad, se tendría que aplicar la funciśn de Makeham al estándar, desagregándolo y después, vía la relación entre logitos: $\hat{\mathrm{Y}}(\mathrm{x})=\alpha+\beta^{\mathrm{S}}(\mathrm{x})$, obtener la tabla de mortalidad por edades individuales.

\section{Conclusiones generales}

Después de haber visto modelos de ajuste, para los fenómenos nupcialidad, fecundidad y mortalidad, se ha resaltado la importancia que 
tiene conocer cuándo un ajuste suaviza la estructura por edad del fenómeno en cuestión, cuándo la corrige y cuándo la caracteriza.

Modelos como los de A. J. Coale, para nupcialidad, el de A.J. Coale y Trussell, para la fecundidad y el sistema logito de W. Brass son modelos que al estar basados en estándares, en el caso de nupcialidad universal y en los casos de fecundidad y mortalidad dependientes de los niveles observados, corrigen las estructuras por edad del fenómeno en cuestión, es decir, los ajustes obtenidos vía los anteriores modelos no necesariamente minimizan los residuales (la diferencia entre los valores observados y los ajustados); esto último dependerá de la calidad de la información con que se cuenta, ya que puede tenerse una estructura por edad poco o muy alejada de la realidad, por lo que no necesariamente se debe esperar un ajuste que se adapte 10 mejor posible a la distribución observada, la cual en general no muestra la situación imperante del fenómeno.

E1 modelo bilogístico de A. Bocaz y la función de fecundidad de W. Brass, sirven para obtener ajustes que suavizan la estructura por edad del fenómeno nupcialidad, en el caso del primero, o fecundidad en el caso del segundo. Los ajustes obtenidos a través de los anteriores modelos no deben ser considerados como ajustes que corrigen la información sino ajustes que suavizan y desagregan la información observada. Ellos no estan basados directamente en estándares sino que se basan en la experiencia observada de dichos fenómenos; la gran ventaja de estos es que sirven para desagregar, a edades individuales, las estructuras por edad tanto de la nupcialidad como de la fecundidad.

Ajustes como los que se obtienen vía la función de Makeham, para el fenómeno mortalidad, caracterizan la distribución por edad de la mortalidad, ya sea a través de las probabilidades de muertes [ $q(x)], 0$ bien de los sobrevivientes a edad $\mathrm{x}[\ell(\mathrm{x})]$. La caracterización se lleva a cabo minimizando los residuales, es decir encontrando una función que se adapte de la manera más adecuada a la experiencia observada, sin que esto quiera decir que se está corrigiendo la información.

Cabe señalar que para los fines de este trabajo una suavización, a diferencia de una caracterización, no necesariamente debe adaptarse; minimizando los residuales, a la estructura por edad observada, lo cual en la caracterización debe buscarse.

Finalmente se recomienda a todos aquellos que utilizan modelos de ajuste que tengan un conocimiento claro del o los modelos que van a aplicar; para ello se debe hacer una evaluación de la calidad de la información con la que se está trabajando, ya que se puede dar el caso que la calidad de la información sea decuada y lo único que requiera sea su desagregación o bien sólo una suavización. Si después 
de evaluar la información se cree necesario corregirla, antes de hacer1o hay que reflexionar sobre los niveles reales que se suponen predominan en la población observada, para con ello seleccionar adecuadamente el estándar o los estándares que servirán en la corrección de dicho fenómeno.

\section{BIBLIOGRAFIA}

Bocaz, A., "Experiencia de nupcialidad por cohortes resumida por un modelo bilogístico", Notas de Población, año VII, No. 19, abril, 1979.

Bocaz, A., "El uso de la ley de Makeham como función demográfica", Notas de Población, CELADE, año II, Vol. 6, diciembre, 1974.

Brass, W., "On the scale of mortality", Biological Aspects of Demography. Taylor and Francis Ltd. London, 1970.

Brass, W., "Seminario sobre métodos para medir variables demográficas (Fecundidad y Mortalidad)", CELADE, Serie DS, No. 9, San José, Costa Rica, 1973.

Coale, A.J. "Age Patterns of Marriage". Population Studies. Vol. XXV, No. 2, Julio, 1971.

Coale, A. J. y T. J. Trussell, "Model Fertility Schedules: variations in the age structure of childbearing in human populations". Populations Index, 40 (2), 1974.

Coale, A. J. y P. Demeny, Regional Model Life Tables and Stable Populations. Princeton, New Jersey, Princeton University Press, 1966.

Coale, A. J. y D. R. Mc.Neil, "The distribution by age of the frequency of first marriage in a female cohort". JASA, Vol. 67, No. 340, 1972.

Retherford, R., "The Brass fertility polinomial", Census forum, Vol. 5, No. 4, 1979. 